\title{
Probabilistic space- and time-interaction modeling of mainshock earthquake rupture occurrence
}

\author{
Luis Ceferino $^{1}$, Anne Kiremidjian ${ }^{1}$, and Gregory Deierlein ${ }^{1}$ \\ ${ }^{1}$ Civil and Environmental Engineering Department, Stanford University
}

May 18, 2021

Corresponding author: Luis Ceferino. E-mail: lceferinor@gmail.com

*This manuscript is a peer-reviewed preprint and has been accepted for publication in the Bulletin of Seismological Society of America. The final version of the manuscript can be found here: https://doi.org/10.1785/0120180220 


\begin{abstract}
This paper presents a probabilistic formulation for modeling earthquake rupture processes of mainshocks. A correlated multivariate Bernoulli distribution is used to model rupture occurrence. The model captures time interaction through the use of Brownian passage-time (BPT) distributions to assess rupture interarrival in multiple sections of the fault, and it also considers spatial interaction through the use of spatial correlograms. The correlograms represents the effect of rupture nucleation and propagation. This model is proposed as an attractive alternative to existing probabilistic models because it (1) incorporates time and space interactions of mainshocks, (2) preserves the marginal distributions of interarrival times after including spatial rupture interactions, i.e., model consistency, and (3) has an implicit physical interpretation aligned with rupture behavior observations. The proposed model is applied to assess the occurrence of large interface earthquakes in the subduction fault along the coast of Lima, Peru. The model matches well both the annual magnitude exceedance rates and the average seismic moment release in the tectonic region. The AIC test confirms that our model performs statistically better than models that do not capture earthquake space interactions. AIC also shows that the spherical correlogram outperforms the exponential correlogram at reproducing earthquake data. Finally, time-dependent seismic hazard in the region is calculated, and the results demonstrate that by accounting for recent earthquake occurrences, the inclusion of time-dependent effects can reduce the 30-year seismic hazard by a factor of four.
\end{abstract}




\section{INTRODUCTION}

Modeling of earthquake rupture occurrence is one of the most important components of earthquake hazard analysis, which underlies Performance-based Earthquake Engineering (PBEE) and earthquake risk assessments. Earthquake rupture modeling remains a particular challenge due to limited data and knowledge to reliably characterize earthquake ruptures. As a result, probabilistic hazard analyses employ multiple simplifying assumptions to account for the interactions between earthquake arrival, location, rupture size, and magnitude.

Two main approaches are used to model earthquake rupture, physics-based and probabilistic. Physicsbased theories have been proposed to explain the underlying mechanics of earthquakes. The elastic rebound theory, first proposed by Reid (1911), describes earthquake ruptures as the result of the sudden releases of elastic strain, which is slowly accumulated through interseismic cycles. Though the elastic rebound theory only provides a conceptual description of the nature of earthquake cycles, it has been the theoretical basis behind more modern, quantitative earthquake theories. Recently, modern rock fracture models and efficient computational tools have allowed physics-based simulations of earthquake rupture cycles (Luo et al., 2017; Richards-Dinger and Dieterich, 2012). At the present, the rate and state friction law (Dieterich, 1979; Ruina, 1983; Marone, 1998) is the canonical model for simulating such cycles. Applications of the state and friction law have allowed researchers to reproduce complex fault rheologies and earthquake rupture behaviors such as nucleation (Galvez et al., 2014), earthquake swarms (Lohman and McGuire, 2007), aftershocks (Dieterich, 1994), postseismic relaxation (Savage and Langbein, 2008), and coseismic, interseismic and postseismic strain and stress cycles (Barbot et al., 2012). While physics-based models are conceptually attractive, they are highly computationally intensive and depend on multiple unobserved fault parameters (e.g., fault normal stresses, constitutive law parameters, fault asperities). These parameters are uncertain, and as a result, propagation of these uncertainties needs to be considered when the seismic hazard is computed. Propagation of parameter uncertainty is extremely challenging due to current computational constraints and to the large sensitivity of the model results to such uncertainties.

Probabilistic formulations for earthquake rupture occurrence have been extensively used for modeling earthquake hazard due to their relative computational simplicity compared to physics-based formulations. Moreover, these models are observational and data-driven and allow for direct incorporation of uncertainty of rupture occurrence. Researchers have proposed several probabilistic models that vary in corresponding physical interpretation, complexity level, and assumed physical interactions over earthquake rupture locations, interarrival times, and magnitudes in a tectonic fault. In spite of their maturity, only few probabilistic formulations have been able to incorporate all these earthquake interactions. Because these interactions are complex, these models have challenging calibration procedures and even introduce model biases into the hazard predictions as described in the model by Field and Gupta (2008) and Field et al. (2015). This paper presents a new probabilistic formulation for modeling earthquake rupture occurrence of mainshocks by explicitly incorporating the modeling of earthquake interactions over time and space, capturing coupling between earthquake rupture locations, interarrival times, and magnitudes. This formulation provides concrete steps forward in probabilistic rupture modeling by (1) including the modeling of key rupture interactions of mainshocks, (2) detailing the physical interpretation of the model, and (3) demonstrating the overcomes the model biases found in Field and Gupta (2008) and Field et al. (2015) both analytically and through simulation.

The paper begins with a brief summary of existing probabilistic models, highlighting their main features and limitations. Then, it describes the proposed probabilistic model including the model's physical interpretation. Next, it provides a case study in the subduction fault along the coast of Lima, Peru, to showcase (1) the applicability of the proposed model and the parameter estimation techniques to replicate the occurrences of large earthquakes in Peru; (2) a benchmarking of the model performance against historical magnitude exceedance rates and spatial distribution of average seismic moment release; (3) a benchmarking of the statistical performance of the model against other probabilistic earthquake models through the Akaike information criterion (AIC); and (4) a comparison of time-dependent earthquake hazard estimates based on the proposed model with the time-independent hazard estimates. A mathematical proof of the theoretical consistency of the model is included in Appendix A, and the formulations of likelihood functions to evaluate AIC are included in Appendix B. 


\section{EXISTING MODELS FOR EARTHQUAKE RUPTURE PRO- CESS MODELING}

Several probabilistic formulations have been proposed to model earthquake rupture occurrence based on either empirical observations or theories of earthquake rupture interactions on seismic faults. The following briefly summarizes four categories of these models.

\section{Time-independent models}

The Poisson model is a time-independent model and is arguably the most frequently used probabilistic model (e.g., seismic hazard data for building codes (Petersen et al. (2014)) given its simplicity in modeling tectonic plate interactions. The canonical Poisson model is homogeneous over a seismic fault, which means that ruptures are equally likely at every point of the fault. Another characteristic of the Poisson model is that the hazard rate, defined as the instantaneous likelihood of earthquake occurrence, is independent of previous earthquakes. This contradicts the elastic rebound theory since it ignores the time-dependent build-up and release of tectonic strains between earthquakes.

\section{Time-interaction models}

Time-interaction models are those that incorporate the earthquake occurrence intervals of characteristic earthquakes. According to the characteristic earthquake theory, seismic faults tend to generate almost the same large rupture with a magnitude in a relatively narrow range close to the maximum (Schwartz and Coppersmith, 1984; Wesnousky, 1994). Though the limited earthquake data has not been able to conclusively support characteristic earthquake models, (Kagan and Jackson, 1991; Geller et al., 2015), their temporal dependencies are based on the elastic rebound theory.

These time-interaction models include temporal dependencies of mainshocks but do not address the spatial dependencies originated from multiple characteristic earthquakes occurring at different fault locations. Additionally, the earthquake interarrival time, i.e., time between subsequent earthquakes, is modeled uncoupled and independent from the rupture magnitude. Probability distributions for earthquake interarrival time included Gaussian (Rikitake, 1974), Weibull (Hagiwara, 1974), Lognormal (Nishenko and Buland, 1987), Gamma (Udias and Rice, 1975), and the Brownian passage-time distribution (BPT) (Kagan and Knopoff, 1987; Matthews et al., 2002). All these models capture the sudden drop of probability of earthquake occurrence immediately after the occurrence of a preceding earthquake. They also capture the probability increase over time as the slip re-accumulates in the seismic fault (Zhuang et al., 2012). These model features correspond to the earthquake behavior described by the elastic-rebound theory. Matthews et al. (2002) performed a thorough comparison among the implied earthquake arrival behavior of the models and developed corresponding mathematical expressions for the their impact on the earthquake hazard rates. Thus, these models capture important characteristics of rupture data, which is consistent with the elastic-rebound theory. In particular, the BPT distribution provides an explicit physics-based representation of the rupture process. As stated by Matthews et al. (2002), the BPT models earthquake ruptures as sudden releases of tectonic stress, which accumulates over interseismic cycles and has a random component.

\section{Time- and magnitude-interaction models}

Time- and magnitude-interaction models are the ones that consider the coupling between the earthquake interarrival time and magnitude. These models build on the slip-predictable and time-predictable hypotheses (Shimazaki and Nakata, 1980). The slip-predictable model (Kiremidjian and Anagnos, 1984) assesses the earthquake interarrival time using one of the time-interaction models described above, and introduces a magnitude function that increases based on the time since the last earthquake. The timepredictable model (Anagnos and Kiremidjian, 1984), on the other hand, assesses the interarrival time with a probabilistic model that is function of the magnitude of the last earthquake. In this model, large earthquake ruptures induce long waiting times for the next earthquake. Similarly, the stress release model assesses the likelihood of earthquake interarrival time as a function of the accumulated tectonic stress, which increases due to tectonic loading and decreases suddenly with each earthquake occurrence (Zheng and Vere-Jones $(1991,1994)$ ). Because the stress drops are calculated using previous earthquake magnitudes with an empirical function, then the stress release model also incorporates interactions between interarrival times and previous earthquake magnitudes. Even though these models incorporate 
coupling between the interarrival time and the earthquake magnitude, they do not address the spatial dependencies originated from earthquake ruptures occurring at different locations of the seismic faults.

\section{Space- and time-interaction models}

Space- and time-interaction models generalize time-interaction models and time- and magnitude-interaction models. Through spatiotemporal interactions, these probabilistic models capture coupling between earthquake interarrival times, locations, and sizes (magnitudes) in a seismic fault. Lutz and Kiremidjian (1995) proposed a pioneering model that coupled such spatiotemporal interactions using a generalized SemiMarkov process. The model discretizes the fault into small sections and evaluates earthquake cycles by (1) selecting the nucleation point according to accumulated slip distribution in the fault sections, (2) estimating the rupture length based on the accumulated slip at the nucleation section, and (3) propagating the rupture to corresponding neighboring sections.

Subsequently, the Uniform California Earthquake Rupture Forecast version 2 (UCERF v2) proposed a different approach composed of two main steps. First, the occurrence probabilities of all possible earthquake ruptures in the discretized fault system are computed using a combination of BPT distributions that incorporate information on the last rupture time in different fault locations (Field and Gupta, 2008; Field et al., 2009). Next, all the rupture occurrences are sampled independently and then combined to estimate the time-dependent seismic hazard. Observations from the model application showed a mismatch between the assumed BPT distributions and the resultant simulated distributions of rupture interarrival times, introducing of model bias in the earthquake occurrence rates and the hazard predictions (Field and Gupta, 2008; Field et al., 2015). This mismatch is not a property of any earthquake observation but a feature of the UCERF model itself. This issue will be referred as lack of model "consistency" hereafter. The UCERF version 3 (UCERF v3) improved the consistency of the model, but it did not achieve full consistency (Field, 2015; Field et al., 2017).

Other methodologies have used smoothed seismicity as spatiotemporal models of earthquake ruptures (Zhuang et al., 2011). Smoothed seismicity models assume that the seismicity rates are the sum of two components: the background seismicity (often heterogeneous in space and stationary in time) and triggered events, e.g., aftershocks (Ogata, 1988; Frankel, 1995; Ogata, 1998; Rhoades and Evison, 2004; Marsan and Lengliné, 2008). Smoothed seismicity models are constructed through kernels, i.e., window functions, that capture variations of seismicity rates over large regions by smoothing the past earthquake locations to infer the spatial distribution of future earthquakes. For example, existing smoothed seismicity models have been proposed to forecast worldwide earthquake rates as a function of location, magnitude, and focal mechanism (Kagan and Jackson, 2014, 2015). Recently, Helmstetter and Werner (2014) proposed a purely data-driven smoothed seismicity model incorporating such spatiotemporal interactions. The model fitted the seismicity data well and gave similar results to those obtained with other models such as the spatiotemporal epidemic-type aftershock sequence (ETAS) (Ogata, 1988, 1998). Because the background seismicity is stationary in time, smoothed seismicity models do not account for interactions of mainshocks over time, and they only can account for triggered events, i.e., aftershocks.

Therefore, very few probabilistic models have been able to capture space and time interactions of earthquake mainshocks. While the two previously described models by Lutz and Kiremidjian (1995) and Field et al. (2015) capture such interactions, these models have been challenging to calibrate due to their complexity and limited data. Such model complexities have introduced bias to the hazard predictions through the previously mentioned lack of model consistency in UCERF (Field et al., 2015). In this paper, we present an alternative earthquake occurrence model to these existing models that incorporates four important characteristics for rupture simulation. (1) The model captures space and time interactions of earthquake mainshocks. (2) The model assumed and simulated distributions of rupture interarrival times are shown to be consistent, in contrast to some other approaches such as the UCERF model. (3) The model uses a simple calibration technique to estimate the model parameters. (4) The model has an implicit physical interpretation compatible with modern earthquake rupture theories. The next sections describe the proposed model, parameter estimation techniques, and the physical interpretation of the model.

\section{PROBABILISTIC MODEL FORMULATION}

The model presented here is the 2-D extension of the 1-D probabilistic rupture model presented by Ceferino et al. (2017). The 1-D probabilistic model was built to assess the spatial and temporal interactions of earthquake mainshock occurrences. The proposed model is based on the fundamental premise of the 
elastic rebound theory, which states that earthquakes are the result of cyclic processes characterized by accumulation of strain and stress over time in a tectonic fault that are released through earthquake ruptures. It follows from this theory that the likelihood of occurrence of new mainshock greatly decreases after the occurrence of such an event and grows as time since the last rupture increases. In faults, seismic activity includes earthquake sequences with aftershocks, foreshocks, and mainshocks clustered in short time windows (Shaw, 1993; Jones, 1994); however, because the aim of the model is to represent the elastic rebound theory and seismic gap effects during long timespans, the paper focuses on mainshocks, which are the events that release the largest amount of slip, energy, and strain in an earthquake sequence.

\section{Notation and representation of the fault and earthquake ruptures}

The model represents the surface of contact, i.e., fault interface, between tectonic plates as an area that is discretized into small sections, for example, Figure 1 shows a fault interface discretized into 15 sections. These sections represent the smallest rupture units, thus they define the lowest earthquake magnitude in the model. The model can represent earthquakes of different sizes in multiple locations of the fault. For instance, Figure 1 shows an earthquake that ruptures nine sections at the central portion of the fault. An earthquake can also rupture all the fault sections, which would trigger the largest earthquake magnitude in the model. The spatial and temporal interactions are confined to the extent of this surface area; therefore, its extent should be selected carefully so that it represents an area isolated from seismic activity in outer regions.

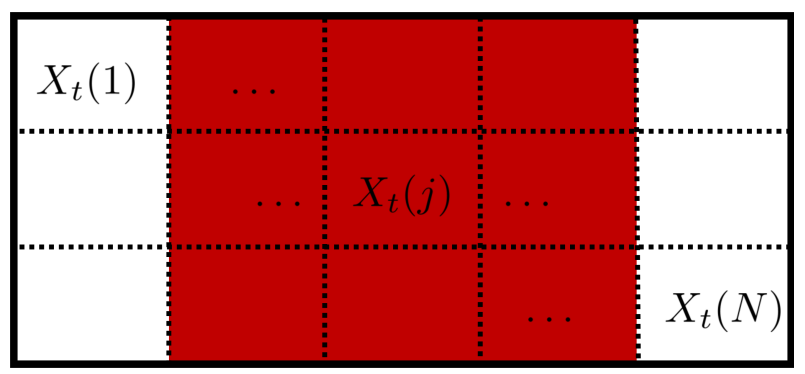

Figure 1: Example of fault discretization with $N=15$ small sections. The 9 shaded sections represent the extent of an earthquake rupture on the fault at time $t$.

Similar to the 1-D version of the model (Ceferino et al., 2017), $\boldsymbol{X}_{\boldsymbol{t}}$ is the rupture vector at year $t$, where $\boldsymbol{X}_{\boldsymbol{t}} \in\{0,1\}^{N}$. N is the total number of fault sections and $t$ is the time index, which is defined to have time steps of one year. Each element of the vector $\boldsymbol{X}_{\boldsymbol{t}}$, shown in Figure 1, is denoted by $X_{t}(j)$ and represents the rupture state of the $j$-th section of the fault. $X_{t}(j)$ is equal to 1 if there is a rupture during year $t$ and 0 otherwise, for $j=1,2, \cdots, N$. Ruptures of multiple adjacent sections in a given year $t$ are assumed to be generated by a single large earthquake event, and ruptures of non-adjacent sections in year $t$ are assumed to be generated by multiple earthquake events. In addition, $\boldsymbol{T}_{\boldsymbol{t}}$ is defined as the vector of the times since the last earthquake until year $t$ for each section, where $\boldsymbol{T}_{\boldsymbol{t}} \in \mathbb{N}^{N}$. $T_{t}(j)$ is the $j$-th element of the vector $T_{t}$ corresponding to the time since the last earthquake in the $j$-th section, where $j=1,2, \cdots N$. Thus, at the next one-year time step: $T_{t+1}(j)$ will either equal $T_{t}(j)+1$ if there was no rupture in the $j$-th section during year $t$ (i.e., $X_{t}(j)=0$ ), or reset to one if there was a rupture (i.e. $X_{t}(j)=1$ ). This relationship is represented in Equation 1.

$$
T_{t+1}(j)=\left(T_{t}(j)\right)\left(1-X_{t}(j)\right)+1
$$

\section{Model description}

The earthquake rupture $\boldsymbol{X}_{\boldsymbol{t}}$ at time $t$ conditioned on the time since the last rupture $\boldsymbol{T}_{\boldsymbol{t}}$ is modeled as a multivariate Bernoulli distribution as shown in Equation 2. The parameters of the multivariate Bernoulli distribution are the rupture occurrence marginal probabilities defined by the vector $\boldsymbol{p}_{\boldsymbol{t}}$ and the rupture occurrence correlations defined by the covariance matrix $\boldsymbol{\Sigma}$. The matrix $\boldsymbol{\Sigma}$ has size $N \times N$ and its elements are based on a spatial correlation model that is explained later in the paper. The vector $\boldsymbol{p}_{\boldsymbol{t}}$ has $N$ elements $p_{t}(j)$, where $p_{t}(j)$ is function of the time since the last rupture $T_{t}(j)$ at the $j$-th section. $p_{t}(j)$ can be estimated as in Equation 3, where $\tau_{j}$ is a random variable that represents the the rupture interarrival time of the $j$-th section. Note that $p_{t}(j)$ is a conditional probability over time of rupture 
occurrence given that there were $T_{t}(j)$ years without a rupture at the $j$-th section. Further, $p_{t}(j)$ is a marginal probability over space, and it does not depend on the years without ruptures in other sections. Then, $p_{t}(j)$ approximates to the annual rupture rate of the $j$-th section for small probability values.

$$
\boldsymbol{X}_{\boldsymbol{t}} \mid \boldsymbol{T}_{\boldsymbol{t}} \sim \text { Mutivariate Bernoulli }\left(\boldsymbol{p}_{\boldsymbol{t}}, \boldsymbol{\Sigma}\right)
$$

$$
p_{t}(j)=P\left[X_{t}(j)=1 \mid T_{t}(j)\right]=P\left[\tau_{j} \leq T_{t}(j) \mid \tau_{j}>T_{t}(j)-1\right]=\frac{P\left[T_{t}(j)-1<\tau_{j} \leq T_{t}(j)\right]}{1-P\left[\tau_{j} \leq T_{t}(j)-1\right]}
$$

The interarrival time $\tau_{j}$ is modeled as a Brownian passage-time (BPT) probability distribution, also known as Inverse Gaussian distribution. The model can also take other distributions (e.g., Lognormal Nishenko and Buland (1987), Gamma (Udias and Rice (1975)), Weibull (Hagiwara (1974))). The model extends the point-source version of the BPT distribution proposed by Matthews et al. (2002) by representing multiple sections of a discretized tectonic fault through the correlated Bernoulli distribution of Equation 2. The BPT model has been widely used in research and applications (e.g. Time-dependent California seismic hazard by Field (2015)). Matthews et al. (2002) gives a comprehensive description of the analysis and applicability of this distribution for modeling earthquake rupture occurrence.

The BPT probability density function (pdf) for $\tau_{j}$ is given in Equation 4. A comprehensive description of the statistical properties of the BPT distribution are provided in Tweedie (1957); Chhikara and Folks (1977). The BPT distribution is defined by the parameters $\mu_{j}$ (mean interarrival time) and $\alpha_{j}$ (aperiodicity or coefficient of variation). The cumulative distribution function (CDF) of the BPT distribution is given in Equation 5, where $\Phi$ is the standard normal CDF. Using Equations 3 and $5, p_{t}(j)$ can be rewritten as in Equation 6. The case study presented in the next section provides descriptions of $p_{t}(j)$ and the BPT distribution.

$$
\begin{gathered}
f_{\tau_{j}}(t)=\left(\frac{\mu_{j}}{2 \pi \alpha_{j} t^{3}}\right)^{1 / 2} \exp \left(-\frac{\left(t-\mu_{j}\right)^{2}}{2 \mu_{j} \alpha_{j}^{2} t}\right) \\
F_{\tau_{j}}(t)=P\left[\tau_{j} \leq t\right]=\Phi\left[u_{1}(t)\right]+e^{2 / \alpha_{j}^{2}} \Phi\left[-u_{2}(t)\right] \\
u_{1}(t)=\alpha_{j}^{-1}\left[t^{1 / 2} \mu_{j}^{-1 / 2}-t^{-1 / 2} \mu_{j}^{1 / 2}\right] \\
u_{2}(t)=\alpha_{j}^{-1}\left[t^{1 / 2} \mu_{j}^{-1 / 2}+t^{-1 / 2} \mu_{j}^{1 / 2}\right] \\
p_{t}(j)=\frac{\left(\Phi\left[u_{1}\left(T_{t}(j)\right)\right]-\Phi\left[u_{1}\left(T_{t}(j)-1\right)\right]\right)+e^{2 / \alpha_{j}^{2}}\left(\Phi\left[-u_{2}\left(T_{t}(j)\right)\right]-\Phi\left[-u_{2}\left(T_{t}(j)-1\right]\right)\right.}{1-\left(\Phi\left[u_{1}\left(T_{t}(j)-1\right)\right]+e^{2 / \alpha_{j}^{2}} \Phi\left[-u_{2}\left(T_{t}(j)-1\right)\right]\right)}
\end{gathered}
$$

The covariance matrix $\boldsymbol{\Sigma}$ contains rupture correlations $\rho_{i, j}$ based on a spatial correlation model. Spatial rupture correlations are introduced represent the influence that a rupture at one section has on triggering a rupture on neighboring sections. In other words, it represents the spatial propagation effect of earthquake ruptures. The correlation is introduced through a correlogram function. Two correlogram functions are evaluated in this paper, the exponential and spherical correlograms. Their respective formulas are given by Equations 7 and 8 . These functions output the correlation $\rho_{i, j}$ of rupture occurrence $X_{t}(i)$ and $X_{t}(j)$ between the sections $i$ and $j$ at any time $t$. The correlation is considered constant over time. The exponential correlogram decays as a function of the distance $\operatorname{dist}(i, j)$ between the $i$-th and $j$-th sections, whereas the spherical correlogram decays as a function of the square of that normalized distance. The parameter $\gamma$ defines the rate at which the correlation decays with distance.

$$
\begin{gathered}
\rho_{i, j}=\exp \left(-\frac{\operatorname{dist}(i, j)}{\gamma}\right) \\
\rho_{i, j}=\exp \left(-\left(\frac{\operatorname{dist}(i, j)}{\gamma}\right)^{2}\right)
\end{gathered}
$$

\section{Approximation through Copula}

Given the probabilities $p_{t}(j)$ and correlations $\rho_{i, j}$ of rupture occurrence, the annual rupture occurrence can be estimated using the correlated multivariate Bernoulli distribution. However, this distribution cannot be written in close-form solution, and therefore sampling from the "true" multivariate correlated 
Bernoulli distribution becomes infeasible. To overcome this issue, the copula method is used, which provides an approximation to the multivariate Bernoulli (Jin et al., 2015). First, a vector $\boldsymbol{Z}_{\boldsymbol{t}}$ of normally distributed random variables is defined. The mean of $\boldsymbol{Z}_{\boldsymbol{t}}$ is a zero-valued vector, and the covariance is represented by the covariance matrix $\boldsymbol{\Sigma}$ of $\boldsymbol{X}_{\boldsymbol{t}} \mid \boldsymbol{T}_{\boldsymbol{t}}$, whose elements come from the correlogram either in Equation 7 or 8 . Then, $X_{t}(j)$ is obtained by evaluating whether $\Phi\left[Z_{t}(j)\right]$ is smaller than $p_{t}(j)$ as in Equation 9, where $Z_{t}(j)$ is the $j$-th element of the vector $Z_{t}$. Because the copula method is approximate, the final spatial correlations of rupture occurrence will be different from the correlation model in Equation 7 or 8. The case study in the paper shows the comparisons between the correlation values from the correlogram models in Equations 7 and 8 and the effective correlations resulting from simulations after applying the copulas method.

$$
X_{t}(j)=1\left\{\Phi\left(Z_{t}(j)\right)<p_{t}(j)\right\}
$$

\section{Physical interpretation of the model}

The probability distribution of the rupture interarrival time defines the rupture process at each fault section. This model uses a BPT distribution for interarrival times because it has a direct underlying physical interpretation of the rupture process (Kagan and Knopoff, 1987). Matthews et al. (2002) described that the BPT distribution models the time of failure occurrence of a Brownian Relaxation Oscillator (BRO). Matthews et al. (2002) interpreted that the BRO behaves as an earthquake rupture process with a load that has two additive components: one represents an increasing load with constant rate, and the other represents a (random) Brownian perturbation. Each time the load hits a fixed threshold, it resets to initial conditions. The "load" in the BRO can represent the accumulated tectonic strain that is released after reaching a strain threshold or the accumulated tectonic shear stress that is released upon reaching a Coulomb stress threshold. Both interpretations represent the earthquake mechanics described in the elastic rebound theory. A direct implication of the BPT and BRO is that the stress gains and thus seismic gaps can increase the likelihood of rupture occurrence. Existing studies have found evidence supporting that stress gains increase the likelihood of earthquake occurrence; for example, Strader and Jackson (2014); Strader et al. (2015) observed that the accumulated static Coulomb stress can indicate future earthquake locations.

Figure 2 illustrates the behavior of a BRO over 500 years. The load threshold was set to 100 load units, with a fixed rate of 1 load unit per year and a Brownian motion with standard deviation of 7 . Each time the load reaches 100 , it resets to 0 . The rupture intearrival time (i.e., time difference between two consecutive threshold hitting times) of this BRO is distributed as a BPT with a $\mu_{j}$ of 100 years and a $\alpha_{j}$ of 0.7 . Similar values of $\mu_{j}$ and $\alpha_{j}$ were found in the application presented later in this paper. A $\mu_{j}$ of 100 years means that the BRO will reach the threshold on average every 100 years. The aperiodicity $\alpha_{j}$ is directly related to the standard deviation (i.e., weight) of the random perturbation in the load path. A 0 -valued aperiodicity would imply fully periodic rupture occurrences with constant interarrival times.

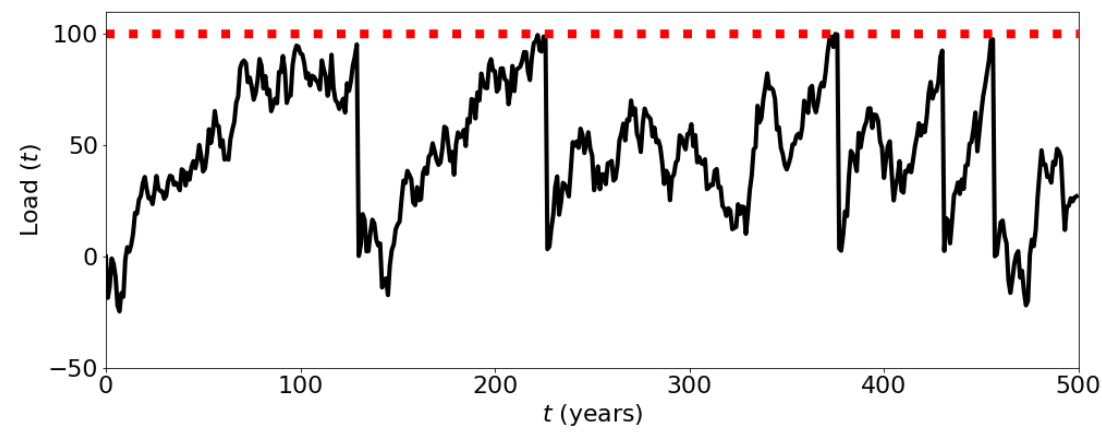

Figure 2: Realization of a load path in Brownian motion for $\mu=100$ years and $\alpha=0.7$ (aperiodicity). The load resets to zero each time it reaches the threshold shown in the dashed line. The load could have units of tectonic stress or shear stress according to the BRO interpretations in Matthews et al. (2002).

The BRO and BPT represent the rupture cycle of each section of the fault, each of which has specific values of mean interarrival $\mu_{j}$ and aperiodicity $\alpha_{j}$. In the model, these BRO's can reach failure simultaneously triggering larger earthquakes (i.e., involving multiple sections). The spatial interactions among 
the BROs at different sections are modeled through a spatial correlation law (e.g., exponential or spherical correlogram). The correlogram introduces an interaction among the stress paths and increases the likelihood of having several neighboring sections reach their respective stress thresholds simultaneously. Figure 3 shows a conceptual representation of three BRO stress paths. The sections can reach failure separately as shown at time $t_{1}$ and $t_{2}$, or fail simultaneously as at time $t_{3}$. The correlation decays with distance in our model; therefore, sections that are at large distances have weak correlation with each other. This model feature can represent the process of rupture nucleation and propagation during large earthquakes since the rupture in one section of the fault can trigger the rupture of neighboring areas of the fault (Ellsworth and Beroza, 1995).

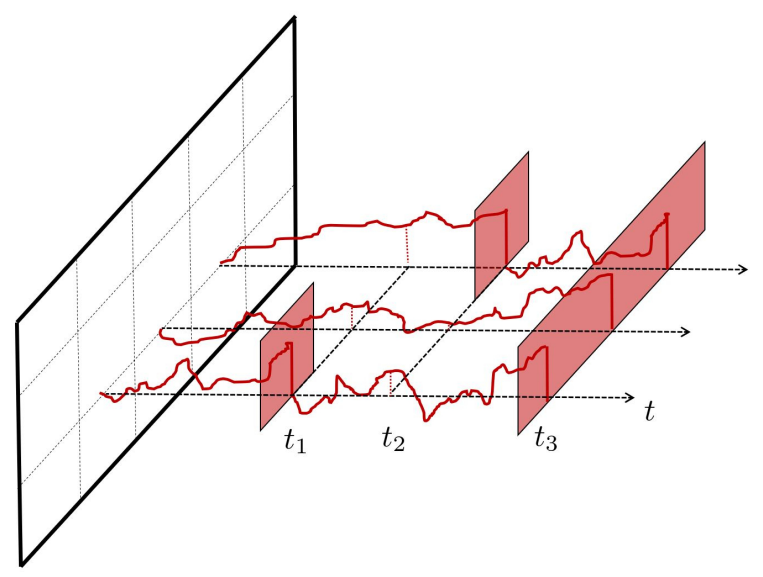

Figure 3: Conceptual representation of stress paths and simultaneous ruptures.

\section{Consistency of the model}

As previously mentioned, some probabilistic models that capture rupture interactions over space and time (e.g., UCERF v2 and UCERF v3) have what are referred to as "consistency" issues. This means that there is a mismatch between the assumed probability distribution of earthquake interarrival time and the resultant distribution from the simulation of earthquake ruptures on a fault (see Field and Gupta (2008); Field (2015)). The proposed model preserves the probability distribution of earthquake interarrival time at each section of the fault after including the spatial rupture interactions. The theoretical proof of this is provided in Appendix A, and the demonstration through simulation is shown later in the case study.

\section{Parameter Estimation}

The model has $2 N+1$ parameters: two for the BPT at each section $\left(\mu_{j}\right.$ and $\left.\alpha_{j}\right)$ and one for the correlogram $(\gamma)$. Performing maximum likelihood estimation (MLE) over all the parameters requires constructing the expression for the joint likelihood of observing the rupture history at all sections of the fault and maximizing this expression to find the parameters' values. Because these procedures are complex, in this paper a simpler approach for parameter estimation is proposed. Instead of performing MLE over the $2 N+1$ parameters of the model, the approach is to 1 ) use MLE to estimate $\mu_{j}$ and $\alpha_{j}$ at each section separately, and then 2) calibrate $\gamma$ in order to match the magnitude exceedance rates and the spatial distribution of the average seismic moment release from historical data. This procedure will be further shown in the case study. Ceferino et al. $(2018,2020)$ provide additional methods for estimating the model parameters that use MLE and Bayesian updating. Such methods are based on a formulation of the likelihood function of the model, a Monte Carlo Markov Chain (MCMC) implementation, and an expansion of the earthquake dataset by leveraging synthetic, physics-based generated earthquake catalogs.

Tweedie (1957) showed that the MLE estimators of $\mu_{j}$ and $\lambda_{j}\left(\lambda_{j}=\mu_{j} / \alpha_{j}^{2}\right)$ can be calculated as in Equations 10 and 11, respectively, where $\tau_{j k}$ are the random samples of interarrival time $\tau_{j 1}, \tau_{j 2}, \ldots, \tau_{j n}$ in the $j$-th section, and $n$ is the total number of samples. Then, $\hat{\alpha}_{j}$ can be estimated as $\left(\hat{\mu}_{j} / \hat{\lambda}_{j}\right)^{-1 / 2}$. 


$$
\begin{gathered}
\hat{\mu}_{j}=\frac{1}{n} \sum_{k=1}^{n} \tau_{j k} \\
\frac{1}{\hat{\lambda}}=\frac{1}{n} \sum_{k=1}^{n}\left(\frac{1}{\tau_{j k}}-\frac{1}{\hat{\mu}_{j}}\right)
\end{gathered}
$$

MLE at individual sections can be improved by incorporating in its log-likelihood function the observed intervals without earthquakes at the beginning and end of the catalog, similarly to Equation 51 by Zhuang et al. (2012).

\section{MODEL APPLICATION TO THE OCCURRENCE OF LARGE INTERFACE EARTHQUAKES NEAR LIMA, PERU}

The model presented in this paper is used to analyze the rupture occurrence of large earthquakes and the associated seismic hazard at the interface (i.e., inter-slab region) of the subduction zone along the Coast of Lima, Peru. The interface surface is the contact area between the South American and Nazca Plates. Figure 4 shows the trench of the subduction zone parallel to the coastline. A detailed description of the tectonic features of this zone can be found in Villegas-Lanza et al. (2016).

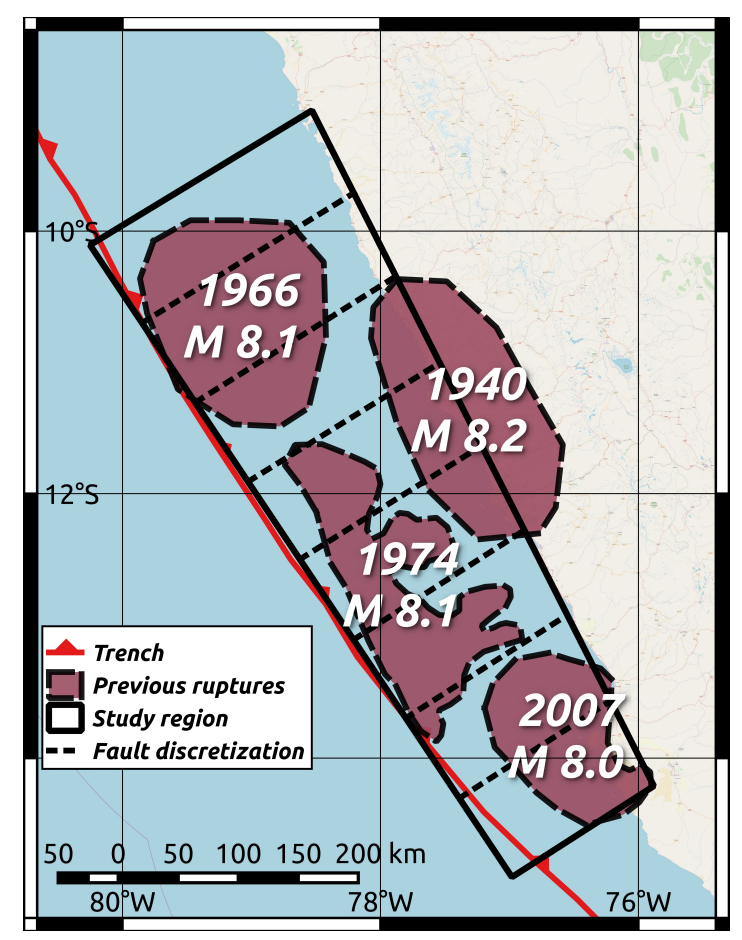

Figure 4: Subduction zone between the Nazca and South American plates. The line parallel to the coastline shows the fault trench, the black quadrilateral shows the region of study subdivided into eight sections, and the four enclosed areas show four earthquake rupture areas of past earthquakes with the year indicated in each area.

\section{Earthquake data}

Rupture data of large earthquakes occurring at the interface of the South American and Nazca Plates are used in this application. The geographic extent of the analysis includes the subduction zone region between the Nazca Ridge (South) and the Mendaña Fracture (North). No previous earthquake has ruptured through these boundaries, and geodetic data shows that there is creeping at these boundaries, i.e., slip locking is negligible (Villegas-Lanza et al., 2016). Therefore, we considered that this geographic extent of the subduction zone is isolated from seismic activity in outer areas. The region of analysis is shown by the large quadrilateral in Figure 4. The length along the strike direction is approximately 
$650 \mathrm{~km}$, and the average width along the dip direction is $190 \mathrm{~km}$. This region was divided into eight sections along the strike direction so that the rupture of an individual section represents an earthquake of magnitude 7.5, the minimum magnitude that the model can represent in this application. Because the region was not subdivided along the dip direction, this application can be considered a 1-D case of the proposed 2-D model.

Figure 4 shows the boundaries between the sections with dashed lines, where each section has a length of approximately $81.3 \mathrm{~km}$. Earthquake data for the region were collected from previous studies. The data contain the last 450 years of seismic activity and include the year of occurrence, the rupture location, and dimensions of earthquake events with magnitude larger than 7.5. Lower magnitudes were incomplete or unavailable for the 450 years, and therefore, they were not included in the analysis. Only interface events were included in the dataset.

The interseismic times between ruptures in different regions of the fault are the key information to calibrate the model. As described earlier, the proposed model represents temporal and spatial interactions of earthquake mainshocks. Accordingly, all the events in our catalog are mainshocks. Dorbath et al. (1990) estimated ruptures dimensions, locations, and magnitudes of earthquake mainshocks based on a collection of damage descriptions and isoseismal maps. The estimates in Dorbath et al. (1990) were used for earthquakes that occurred before 1940, prior to the installation of seismic recording stations. Data about earthquake mainshocks occurring after 1940 were collected from different sources, including (1) the rupture areas and locations of the 1940 and 1966 earthquakes estimated by Kelleher (1972), (2) the magnitudes of the 1940 and 1966 earthquakes estimated by Kanamori (1977), and (3) the rupture dimensions, locations, and magnitudes of the 1974 and 2007 earthquakes estimated by Langer and Spence (1995), and Chlieh et al. (2011), respectively. The geographic data of the earthquake rupture set were georeferenced and compiled in a geographic information system (GIS) file and are in a link in the Data and Resources section. Figure 4 shows the rupture areas of the 1966, 1940, 1974, and 2007 earthquakes. Table 1 shows list of total earthquakes and corresponding magnitudes in the historical catalog.

The information about the earthquake mainshocks in the study region was gathered from existing studies. However, applications of the proposed model to other tectonic faults might require to decluster the catalog in order to isolate the earthquake mainshocks from the aftershocks. Existing declustering methods can be used for this purpose, e.g., Gardner and Knopoff (1974); Reasenberg (1985); Marsan and Lengliné (2008). Additionally, our catalog did not have complex earthquake sequences such as doublets: two closely located mainshocks that occur with minutes to weeks of temporal separation (Kagan and Jackson, 1999). Yet, applications to other tectonic faults might encounter such events, for example, the New Guinea trench off the coast of Indonesia had an earthquake doublet in 2009 with magnitudes of 7.6 and 7.4 occurring three hours apart from each other (Poiata et al., 2010). Because the proposed model aims to represent seismic gap effects during long timespans rather than this short-tterm complex rupture behavior, for modeling purposes, we suggest lumping earthquake doublets into one larger earthquake with equivalent aggregated moment release and rupture area. As a result, interseismic times will not be shorter than the proposed temporal resolution of 1 year and the long-term energy releases in the plate still will be captured by the model.

Table 1: Magnitude and occurrence year of historical earthquakes in the last 450 years.

\begin{tabular}{|c|c||c|c|}
\hline Mw & Year & Mw & Year \\
\hline 8.1 & 1586 & 8.6 & 1746 \\
7.5 & 1664 & 8.2 & 1940 \\
7.85 & 1678 & 8.1 & 1966 \\
8.4 & 1687 & 8.1 & 1974 \\
7.5 & 1725 & 8.0 & 2007 \\
\hline
\end{tabular}

The tectonic region in our case study was divided into sections with spatial dimensions that are able to generate the minimum earthquake magnitude in the catalog, which is 7.5 (Table 1). This earthquake magnitude has a rupture length of approximately $81 \mathrm{~km}$ according to the scaling law proposed by Strasser et al. (2010). Because the region has approximately $650 \mathrm{~km}$ along the strike direction, it was divided into eight sections of equal length along this direction as shown in Figure 4. Coarser subdivisions of the tectonic plate would not allow the model to reproduce the rupture area corresponding to the minimum magnitude. In contrast, finer subdivisions would enable the model to represent smaller rupture areas, and thus lower earthquake magnitudes. Additionally, finer subdivisions would enable the model to better capture the location of earthquake occurrence. However, the available data are sparse and limited to 
magnitudes larger than 7.5, therefore, the data are not suitable for performing further reductions on fault section dimensions. Thus, we recommend that model implementations utilize fault section sizes corresponding to the rupture area of the minimum magnitude in the historical catalog of interest.

The ruptures were projected along the trench of the subduction zone, allowing this case study to be represented in one dimension. Figure shows the rupture projections along the strike direction over time from South to North. The rupture lines were discretized and associated to individual sections of the fault. Rupture segments smaller than half of the sections' lengths were considered negligible. The resultant rupture lengths are shown in Figure. Table 2 summarizes the 450 -year catalog and shows the interarrival times and the time since the last rupture at each section of the fault.
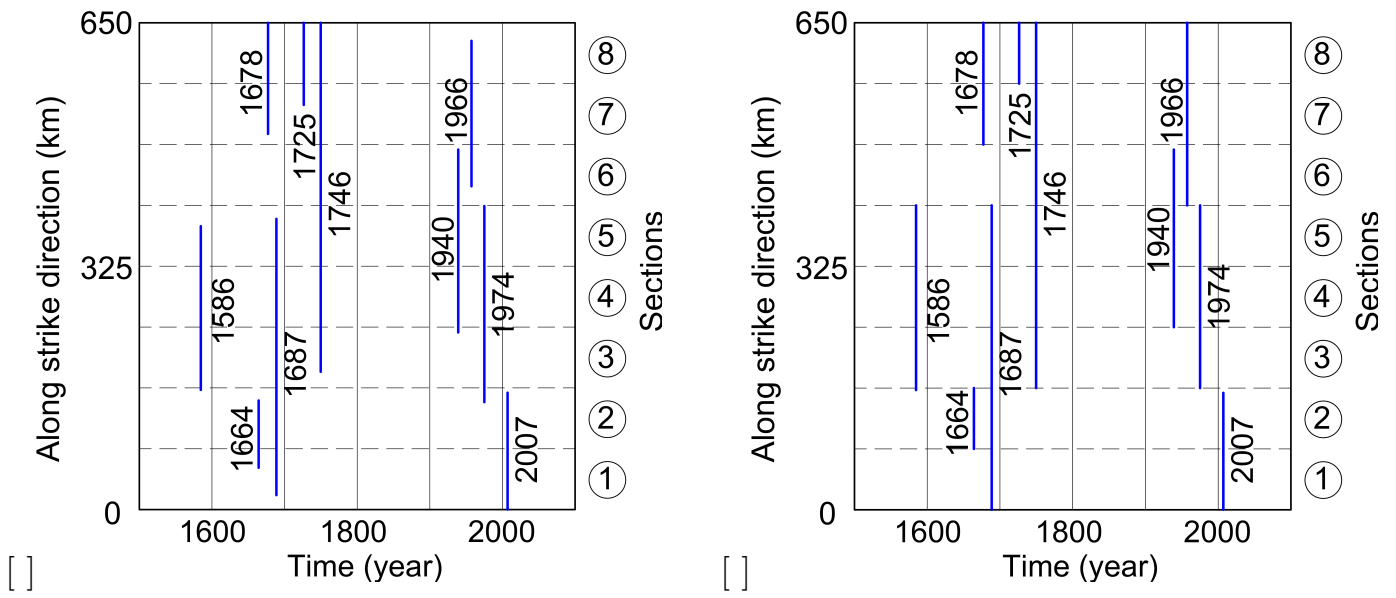

Figure 5: Projections of earthquake ruptures to the trench of the subduction zones over time: (a) estimated rupture lengths, (b) discretized rupture lengths.

\section{Parameter estimation}

The 450-year catalog was used to estimate the 17 parameters of the model (i.e., $\mu_{j}$ and $\alpha_{j}$ in each section and $\gamma$ ). As described previously, the parameter estimation is performed in a two-step process. First, the BPT parameters $\mu_{j}$ and $\alpha_{j}$ at each section are evaluated, and then, the correlation parameter is estimated. Other techniques for parameter estimation can be found in Ceferino et al. (2020), and a comparison of the effect of the parameter estimation technique selection on the seismic hazard can be found in Ceferino et al. (2018).

\section{Parameter estimation for individual sections}

The parameters $\mu_{j}$ and $\alpha_{j}$ are estimated at each fault section separately using Equations 10 and 11. Table 2 shows the estimated $\mu_{j}$ and $\alpha_{j}$ in each section. Because the sparsity of the data did not allowed a more reliable parameter estimation, two adjustments to the estimated parameters were made. The first adjustment was to the estimates of aperiodicity $\alpha_{j}$ (i.e., coefficient of variation) in sections 2,6 , and 8 of the fault. The estimates of aperiodicity $\alpha_{2}, \alpha_{6}$ and $\alpha_{8}$ were considered unreliable since they were large compared to the aperiodicity in other sections and were particularly more sensitive to the data sparsity of our case study. Therefore, a value of 0.7 was assigned to the $\alpha_{j}$ of these three sections since 0.7 is closer to the values found in sections with more data points and is also closer to Bayesian estimates in a similar case study in the region (Ceferino et al., 2018). The second adjustment was to the estimates in section 1 . The $\mu_{1}$ and $\alpha_{1}$ values of section 1 were assumed to be equal to those of section 2 because there were not enough data points in section 1 to perform parameter estimation.

Figure shows the BPT distribution in black for sections 4 and 5 using the values of $\mu_{j}$ and $\alpha_{j}$, calculated previously. Because all the ruptures in sections 4 and 5 occurred due to the same earthquake events, the interarrival data and the estimated parameters $\mu_{j}$ and $\alpha_{j}$ are the same for both sections. In addition, Figure shows an exponential distribution with the equivalent mean of interarrival time. The exponential distributions of interarrival time are derived from the Poisson model and are used to model time-independent earthquake hazard. The graph shows that that the exponential distribution has higher 
Table 2: Earthquake data and estimated parameters for all sections using Equations 10 and 11 for MLE at individual sections.

\begin{tabular}{|l|cccccccc|}
\hline & \multicolumn{7}{|c}{ Section labels } \\
& 1 & 2 & 3 & 4 & 5 & 6 & 7 & 8 \\
\hline Last rupture occ. (year) & 2007 & 2007 & 1974 & 1974 & 1974 & 1966 & 1966 & 1966 \\
\hline Interarrival times (years) & 320 & 320 & 228 & 34 & 34 & 26 & 220 & 220 \\
& & 23 & 59 & 194 & 194 & 194 & 68 & 21 \\
& & & 101 & 59 & 59 & & & 47 \\
& & & & 101 & 101 & & & \\
\hline$\mu$ (years) (MLE) & $(\star)$ & 172 & 129 & 97 & 97 & 110 & 144 & 96 \\
$\alpha$ (MLE) & $(\star)$ & $1.73^{(\star)}$ & 0.59 & 0.70 & 0.70 & $1.18^{(\star)}$ & 0.62 & $1.16^{(\star)}$ \\
\hline
\end{tabular}

* Note that for simulation with the earthquake model, a further adjustment to the parameters was conducted in sections with little data or sensitive estimates. $\alpha_{2}, \alpha_{6}$ and $\alpha_{8}$ were set to 0.7 , and $\mu_{1}$ and $\alpha_{1}$ were set equal to $\mu_{2}$ and $\alpha_{2}$.

probability of small interarrival times than the BPT distribution. The time-independent exponential model will be used later in the paper to provide comparisons with the presented model.

Figure shows the annual rupture probability $p_{t}(j)$, as a function of $T_{t}(j)$, and the number of years since the last rupture at the $j$-th section, for fault sections 4 and 5 using the BPT and exponential interarrival time models. $p_{t}(j)$ was defined as the probability of occurrence of an event in the next one-year time increment given that there were $T_{t}(j)$ years without an earthquake in the section. Thus, $p_{t}(j)$ is equivalent to the definition of earthquake occurrence hazard rate for time increments of one year. Figure 6 shows that $p_{t}(j)$ starts from 0 , increases up to a maximum point, and then flattens out in all the sections of the fault. Chhikara and Folks (1977) proved mathematically that for any value of $\mu_{j}$ and $\alpha_{j}$, the rate $p_{t}(j)$ starts at 0 , increases to a maximum value, and then decreases until reaching a constant asymptotic level. The BPT implies that an earthquake rupture at the $j$-th section immediately unloads all the stress at the corresponding location since the failure rate decreases to 0 after an event. In the BPT model, the earthquake occurrence probability saturates to a constant value after a long seismic gap. This saturation suggests that, after a long period of time, all the additional tectonic stress is released through creep or other means so that the hazard rate is not increased (Matthews et al., 2002). Although such a saturation has not been corroborated from empirical observations, it remains a major consequence of the BPT model. Figure also shows the time-independent rupture probability rates resulting from the equivalent exponential pdf shown in Figure. It can be seen that after an earthquake, the BPT rates start estimating rupture probabilities below the time-independent estimation. Then, because the timeindependent rate remains constant over time, the BPT rates increase to higher probability levels. This feature is key to explaining the differences between thee time-dependent and independent estimations of seismic hazard in Lima.
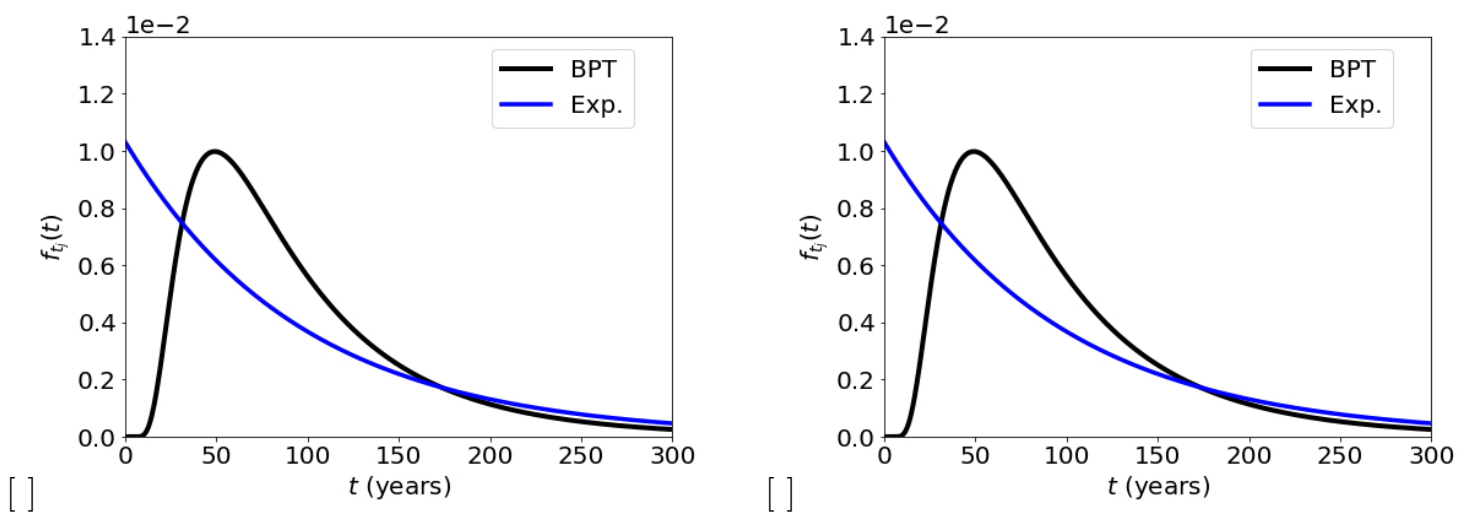

Figure 6: BPT in sections 4 and 5 in black and exponential with equivalent mean in lighter color: (a) probability density function, (b) hazard rate. 


\section{Parameter estimation for the correlogram}

The parameter $\gamma$ is calibrated for the exponential and the spherical correlation models. Multiple realizations of rupture occurrence were sampled using different values of $\gamma$. In this calibration, rupture occurrences in a time horizon of 500,000 years were analyzed. The earthquake ruptures were simulated for each year in the time horizon in a sequential manner using Equation 2. The simulation starts at the year 2018 based on the numbers of years since the last rupture at each section as indicated by the historical catalog in Figure 5. At each year in the simulation, the years since the last rupture at each section were adjusted according to the generated earthquakes in the simulation.

The scaling equations for interface subduction-zone earthquakes, proposed by Strasser et al. (2010), were used to relate the rupture length in the sections to earthquake magnitude. Although the rupture length to magnitude relationship was treated as deterministic, the uncertainty in this relationship can be incorporated in the model. The model can generate a maximum earthquake magnitude of 8.8 as a result of the simultaneous rupture of all the sections (i.e., rupture length of $650 \mathrm{~km}$ ). The annual magnitude exceedance rates and the average annual seismic moment release were calculated using these realizations for multiple values of $\gamma$. Additionally, magnitude exceedance rates and seismic moment releases were computed using the 10 earthquakes in the historical catalog in Figure 5. The $\gamma$ values that generated occurrences closely matching the results from the historical data were selected. The scripts used for the calibration procedure are provided in a link the Data and Resources section.

The exponential correlation model in Equation 7 was tested using multiple $\gamma$ values. This paper describes the results for $\gamma$ values equal to $600,900,1,200,1,500$, and 1,800 km. Figure shows a snapshot of the first 1,500 years of rupture simulation for $\gamma$ equal to $1,200 \mathrm{~km}$. The horizontal axis represents the years of earthquake occurrence, and the vertical axis represents the distance along the strike direction form South to North. The past earthquake observations are shown in black, and the simulated ruptures are shown in a lighter color. In the 500,000-year simulation, we observed a few years with simulated ruptures involving non-contiguous fault sections. Because non-contiguous sections are separated by at least $81.3 \mathrm{~km}$, i.e., sections' length, such ruptures were treated as different mainshocks occurring at the given year.
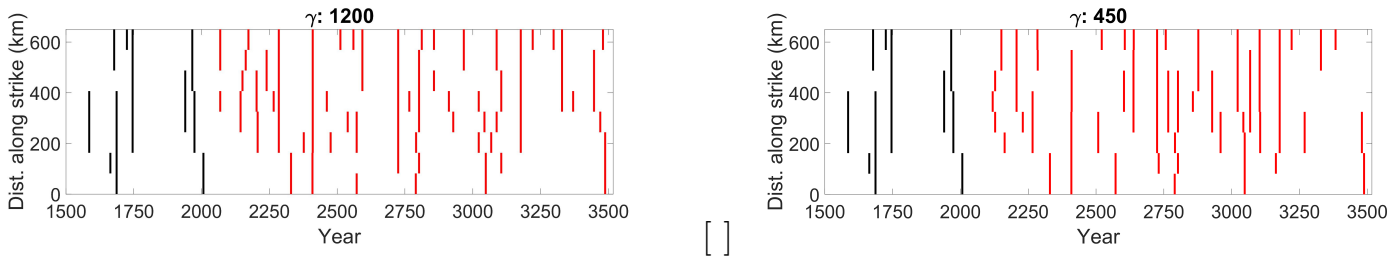

Figure 7: 1500-year rupture simulation ( $\gamma$ values are in $\mathrm{km}$ ): (a) exponential correlogram, (b) spherical correlogram.

Figure shows the exponential correlogram for the five values of $\gamma$. The graph shows the distance between the sections in the horizontal axis and the correlation between two sections in the vertical axis. The correlation starts at 1 when the distance is $0 \mathrm{~km}$, and it decreases as a function of distance. Larger values of $\gamma$ correspond to slower decreases of the correlation at large distances. Note that the correlogram is used to model the correlation on the $\boldsymbol{Z}_{\boldsymbol{t}}$ vector (from the copulas approximation) and not the correlation on the $\boldsymbol{X}_{t}$ rupture vector. Therefore, the exponential correlogram does not match the correlations on $\boldsymbol{X}_{\boldsymbol{t}}$. In this paper, the correlations on $\boldsymbol{Z}_{\boldsymbol{t}}$ are referred to as "apparent" correlations, whereas the correlations on $\boldsymbol{X}_{\boldsymbol{t}}$ are referred to as "effective" correlations.

To analyze the effective correlations, the rupture correlations of section $X_{t}(1)$ with each of the other sections were calculated using the 500,000-year simulations. The effective correlations are also shown in Figure for each of the $\gamma$ values. The contrast between the apparent and effective correlation show that the copula method reduces the correlation values from the correlogram. Although the difference between the apparent and effective values of correlation does not change the selection of the $\gamma$ value, it is important to note that the final rupture correlations introduced to the model are smaller than the values of the exponential correlogram. Additionally, Figure shows the rupture correlations of $X_{t}(1)$ with other sections estimated from the earthquakes in the historical catalog. The comparison shows that for short distances (less than $150 \mathrm{~km}$ ), the effective correlations in the exponential model are lower than the correlation found with data. The comparison also shows that for long distances (more than $300 \mathrm{~km}$ ), the correlations found from data decay faster than the effective correlations with the exponential model.

The lines in Figure depict the annual magnitude exceedance rates resulting from the 500,000 years 

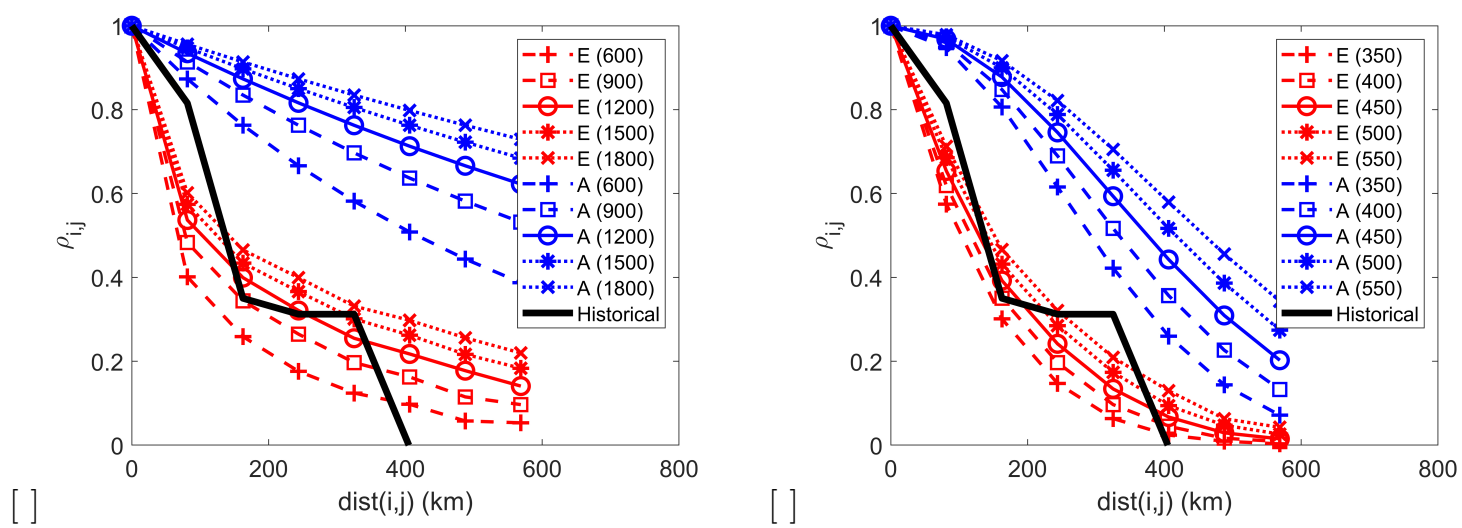

Figure 8: Apparent (A), effective (E), and data-driven (Historical) correlations using different $\gamma$ values (in parenthesis in $\mathrm{km}$ ): (a) exponential correlogram, (b) spherical correlogram.

of rupture simulation using the exponential correlogram with the five $\gamma$ values shown in Figure . Figure also shows the magnitude exceedance rates from the earthquake catalog. The results of the five $\gamma$ values show a good match to the exceedance rates from the data for magnitudes between 7.5 and 8.4. The model outputs fast-decreasing rates for larger magnitudes. As mentioned previously, the maximum magnitude was considered to be 8.8 as the result of a rupture over the eight fault sections. The catalog has only one earthquake larger than 8.4 (i.e., only the $1746 \mathrm{Mw} 8.6$ earthquake). Therefore, conclusive comparisons of empirical Mw rates with the model beyond $\mathrm{Mw} 8.4$ are not possible. Larger values of $\gamma$ increase the rates of large earthquakes because high $\gamma$ values generate stronger effective correlations and therefore higher likelihood of rupturing multiple sections simultaneously.

Figure depicts the average yearly release of seismic moment in the sections of the fault for the five values of $\gamma$. The average release obtained from the earthquake data is shown by the solid black line. Higher $\gamma$ 's had larger associated average moment release because $\gamma$ makes more likely the occurrence of larger earthquakes. Though the rates from all different values of $\gamma$ gave comparable results, $\gamma$ equal to $1,200 \mathrm{~km}$ gave the best match.
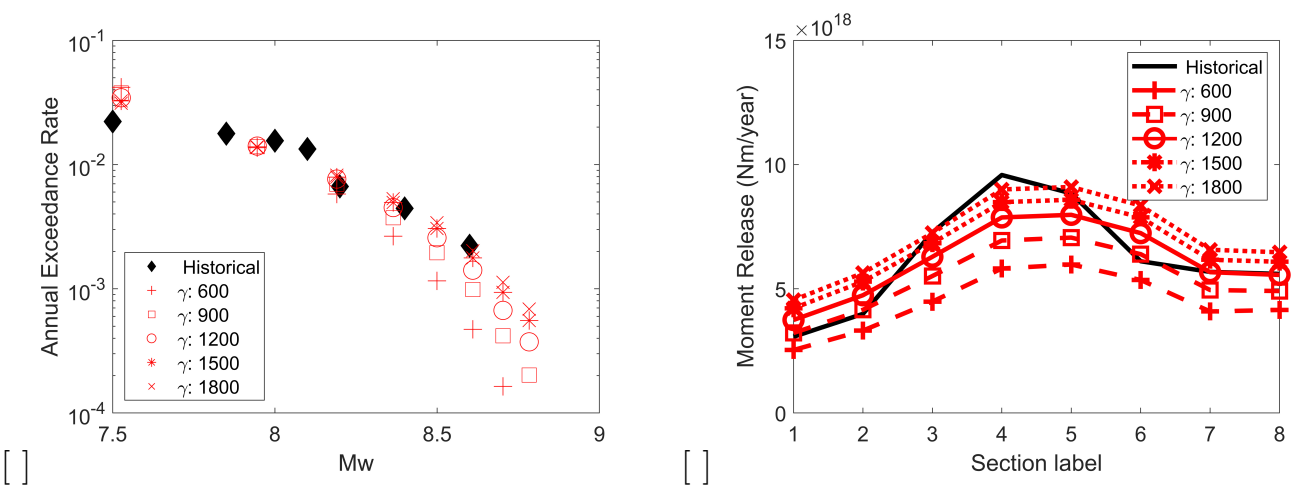

Figure 9: Model benchmark for different exponential correlograms: (a) Magnitude exceedance rates, (b) Average seismic moment (Nm/year).

The spherical correlation model in Equation 8 was also tested using multiple $\gamma$ values. This paper shows the results for $\gamma$ values equal to $350,400,450,500$, and $550 \mathrm{~km}$ because they provided good match to earthquake data. Figure shows a snapshot of the first 1,500 years of rupture simulation for $\gamma$ equal to $450 \mathrm{~km}$. We also observed that a few ruptures involved non-contiguous sections in the 500,00-year simulation, however, such ruptures were less frequently than with the exponential model because the spherical model has spatial rupture correlations that decay faster for large distances.

Figure shows the spherical correlation model for the five $\gamma$ values. Larger values of $\gamma$ correspond to higher values of correlation. These spherical correlations are slightly higher at short distances than the exponential correlations but lower for long distances (starting from around 150 to $200 \mathrm{~km}$ ). Similar to the case of the exponential correlogram, the spherical correlogram does not show the effective correlation values of the rupture vector because the copulas method is used. The effective correlations of the rupture 
in section $X_{t}(1)$ and the ruptures in each of the other sections were calculated from the 500,000-year simulation, and they are shown in Figure. The effective correlations from the spherical correlogram are also slightly higher for short distances than the ones from the exponential correlogram, but they are lower for long distances. The spatial correlations found with the historical catalog match better the effective correlations from the spherical correlogram for both short and long distances than the ones from the exponential correlogram. However, historical catalogs with longer durations are needed to find the empirical spatial correlations more robustly and support the selection of a particular correlation model.

Figure depicts the annual exceedance rates resulting from the 500,000-year simulation with the spherical correlogram using the five $\gamma$ values shown in Figure. The black dots show the exceedance rates corresponding to the earthquake catalog. All the five $\gamma$ values also show a good match to the exceedance rates from the data for magnitudes between 7.5 and 8.4. The annual rates for large magnitudes saturate at a faster rate than when using the exponential correlogram because the spherical correlogram only sustains smaller effective correlations at large distances, as shown in Figure 8.

Figure shows the average annual release of seismic moment for the five values of $\gamma$. The black, solid line represents the average release obtained from the earthquake data. There is a direct relation between larger values of $\gamma$ and larger releases of seismic moment. A $\gamma$ equal to $450 \mathrm{~km}$ gave the results that best approximate the moments obtained from the earthquake data.
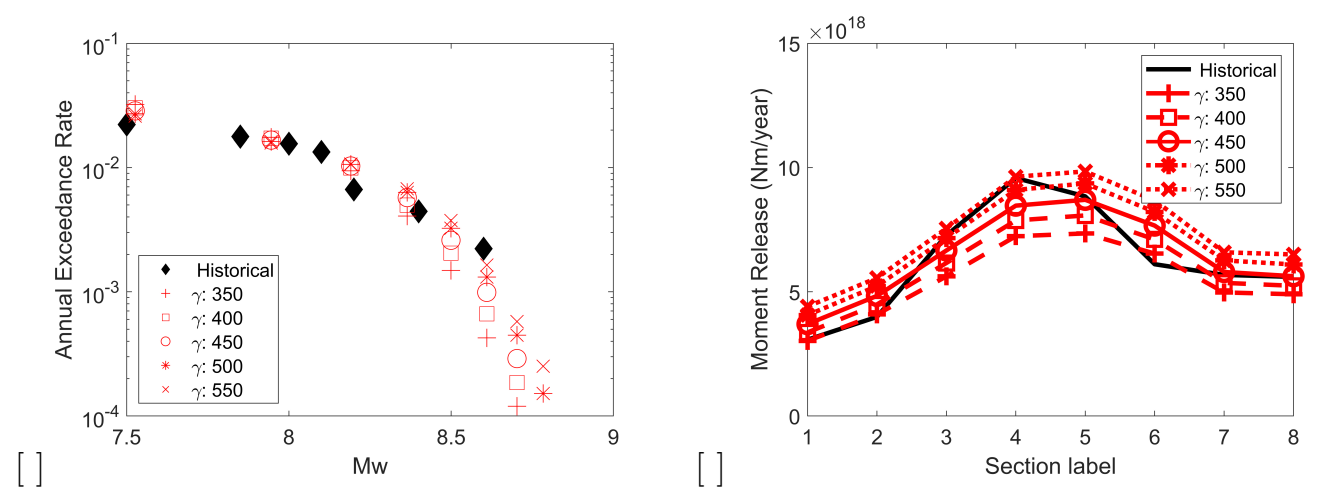

Figure 10: Model benchmark for different spherical correlograms: (a) Magnitude exceedance rates, (b) Average seismic moment (Nm/year)

In summary, both the exponential and spherical correlograms closely match both the magnitude exceedance rates and the annual seismic moment, however, the spherical correlogram performs better at matching the empirical rupture correlations. The best fit to the seismic data with the exponential correlogram was achieved with a $\gamma$ value of $1,400 \mathrm{~km}$, and the best fit to the data with the spherical correlogram was achieved with a $\gamma$ value of $450 \mathrm{~km}$.

\section{Model consistency through simulation}

The model was next tested for consistency. For the model to be consistent, the initially assumed BPT distribution at each section should match the interarrival distribution resulting from the simulation process, after including the spatial interactions among multiple sections. The mathematical proof of model consistency is provided in Appendix A. Here, the distribution of interarrival times from simulation showing consistency in section 4 with $\gamma$ equal to $450 \mathrm{~km}$ is displayed in Figure 11. Similar plots showing consistency for all other sections and for a wide range of $\gamma$ values can be found in the Data and Resources section. The back curve shows the initial BPT as defined in section 4 (i.e., $\mu_{4}=97$ and $\alpha_{4}=0.7$ ), and the bars depict the normalized histogram of rupture interarrivals resulting from the 500,000 years of simulation. The 5,064 ruptures occurred in section 4 during the simulation. As the plot shows, the simulation confirms that the histogram approaches the initial BPT distribution of section 4 . The same procedure was applied to the other fault sections, and the results confirm that the histograms approached the respective BPT distributions in all the other fault sections. Therefore, the simulations demonstrate that the model has consistency. 


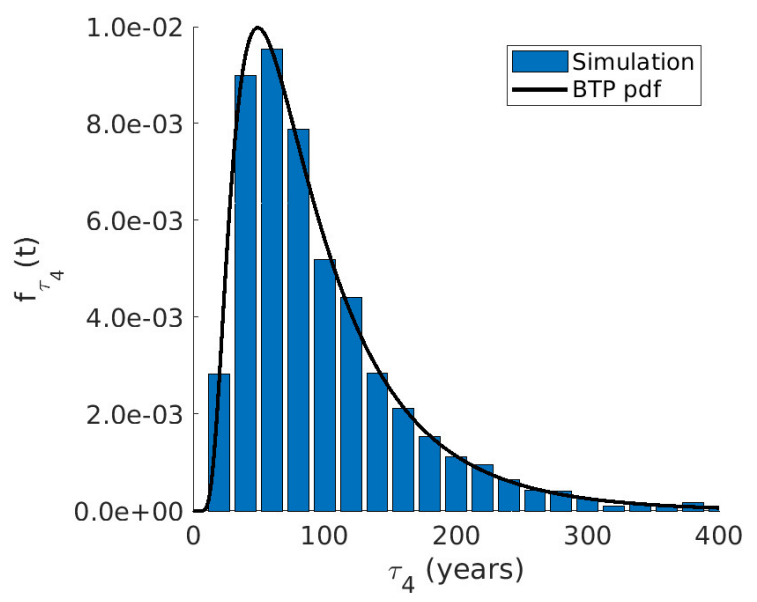

Figure 11: Demonstration of model consistency through simulation. BPT pdf in section 4: Initially assumed vs. simulation after including spatial interactions. In this simulation, the correlation length $\gamma$ $450 \mathrm{~km}$

\section{Statistical Performance of the Model}

The Akaike information criterion (AIC) was used to evaluate the ability of the proposed model to reproduce space and time interactions of large earthquakes. AIC is an estimator that measures the relative quality of statistical models to represent the process that generate data (Akaike, 1974). The AIC value is estimated as shown in Equation 12, where $p$ is the number of parameters in the model, and $\hat{L}$ is the likelihood of observing a dataset according to a given statistical model.

$$
A I C=2 p-2 \ln (\hat{L})
$$

Statistical models with lower AIC values are preferred over models with higher values because the former increase the likelihood of reproducing a dataset with fewer model parameters. This trade-off between goodness of fit and model simplicity balances the risks of overfitting and underfitting data (Bozdogan, 1987).

We use AIC to evaluate whether the proposed model is more suitable than a simplified model to reproduce the earthquake data in Peru. For comparison purposes, we constructed the simplified model by incorporating time interactions but not space interactions. The simplified model uses a single BPT distribution to evaluate earthquake interarrival times thorugh the entire fault in Figure 4 . In contrast to the proposed model, which has $N$ sets of parameters $\mu_{j}$ and $\alpha_{j}$ to capture interarrival times, the simplified model has only a single set of parameters $\mu$ and $\alpha$. Additionally, the simplified model uses an double-truncated exponential magnitude distribution parametrized by $\beta$ (Utsu, 1969; Cosentino et al., 1977), with 7.5 and 8.8 as minimum and maximum magnitudes in the fault, which are the magnitude bounds of our proposed model. Finally, the simplified model assumes that earthquake locations have a uniform distribution through the entire fault. Thus, only 3 parameters define the simplified model: $\mu, \alpha$ and $\beta$.

The parameters $\mu$ and $\alpha$ of the simplified model were estimated using the data points from Table 1 and the Equations 10 and 11. The magnitude distribution parameter $\beta$ was estimated according to Cosentino et al. (1977). Because the earthquake location distribution is uniform over the fault, it did not required further calibration.

The proposed model has 17 parameters in our case study. The parameters $\mu_{j}$ and $\alpha_{j}$ at each fault section were taken from the previous calibration. The parameter $\gamma$ for the exponential correlogram was set to $1200 \mathrm{~km}$, whereas $\gamma$ was set to $500 \mathrm{~km}$ for the spherical correlogram because they fitted well the earthquake data (Figure 9 and 10).

Next, we evaluated the AIC values for three models: the simplified model and the proposed model with exponential and spherical correlograms. The likelihood functions $\hat{L}$ for each model are formulated in Appendix B. The final AIC values are shown in Table 3. These results show that the proposed model with either the exponential or the spherical correlogram performs better than the simplified model. Even though our model has more parameters than the simplified model, it increases significantly the probability of observing earthquake data, balancing the risk of overfitting and effectively incorporating 
space-time interactions that the simplified model does not capture. The AIC values also show that proposed model with spherical correlogram performs better than with the exponential correlogram. Because the exponential correlogram sustains higher correlations at longer distances than the spherical correlogram, these AIC values indicate that the earthquake data are better represented by a faster decay in the rupture correlations, with effective correlations rapidly decreasing below 0.4 for distances larger than $200 \mathrm{~km}$ (Figure 8).

Table 3: AIC values for different probabilistic earthquake models. Lower AIC values indicate better model performance.

\begin{tabular}{|l|c|}
\hline Model & AIC \\
\hline Simplified model & 235 \\
Proposed with exponential correlogram & 208 \\
Proposed with spherical correlogram & 198 \\
\hline
\end{tabular}

\section{Comparison of time-dependent and independent hazards}

This model was also used to estimate the time-dependent seismic hazard in the region and to compare the results with the time-independent seismic hazard. The spherical correlogram was utilized because it performed better than the exponential correlogram in the AIC test, and $\gamma$ equal to $450 \mathrm{~km}$ was used because it matched well the annual exceedance rates and seismic moment releases of the earthquake data, as stated previously. The hazard analysis includes only the contribution of magnitudes larger than 7.5 in the tectonic region. The extent of the simulated earthquake areas was taken from the area covered by all the fault sections the each simulated earthquake ruptures (Figure 1). Abrahamson et al. (2016) developed a ground motion prediction equation (GMPE) for interface earthquakes in subduction zones. This GMPE was used to estimate peak ground accelerations (PGA) in the region. The hazard was calculated using OpenQuake software (Silva et al., 2014), assuming a soil type B (Vs30 = 760 m/s) through the entire region of analysis.

The time-independent hazard was estimated using a double-truncated exponential fitted according to Cosentino et al. (1977). As mentioned previously, the minimum Mw was taken as 7.5, and the maximum as 8.8, which is equivalent to the rupture of all the eight sections in the fault. Additionally, the probabilities of earthquake occurrence were considered uniform over the tectonic region of analysis, which is represented by the in black polygon in Figure 12. Figure shows the time-independent probabilities of exceeding $0.4 \mathrm{~g}$ of PGA in 30 years. Two features are notable from the graph. First, the hazard decreases with distance from the tectonic region because the ground shaking attenuates with distance from the rupture. Second, the time-independent seismic hazard close to the mid zone of the tectonic region is larger than the hazard close to the northern and southern boundaries. Site locations near the middle of the fault zone are exposed to larger number of events than those near the ends of the fault. The mid zone is exposed to strong shaking from mid- and large-size earthquakes that originate at both the northern and southern boundaries, whereas, say, the southern end of the fault is exposed to weak shaking from earthquakes originating at the northern end.

To estimate the time-dependent hazard, a Monte-Carlo simulation was performed on the proposed model. The probabilities of earthquake occurrence during the next 30 years were estimated by analyzing every feasible rupture in sections of the fault model. Because the fault model has eight sections, there are 36 feasible earthquake ruptures: eight events rupturing single sections, seven rupturing two adjacent sections, six rupturing three adjacent sections, and so on. In general, the number of feasible earthquakes can be estimated as $N \times(N+1) / 2$. For each feasible earthquake, the shaking distribution was calculated according to the GMPE by Abrahamson et al. (2016). The probabilities of exceeding a PGA of $0.4 \mathrm{~g}$ during the next 30 years (from 2018 to 2047) were estimated over the region using both the probabilities of earthquake occurrence from the Monte Carlo simulation and the respective shaking distributions.

Figure shows the ratio between the resulting time-dependent probabilities and those from the timeindependent analysis in Figure. In most of the central and northern regions, the time-dependent analysis results in similar hazard predictions $( \pm 25 \%)$ to the time-independent hazard. Between 2018 and 2047 , most fault sections in the central and northern regions will reach interarrival times with rupture rates that are similar to the corresponding time-independent exponential distributions (see Figure ), leading to such similar hazard predictions. However, in the southern region, the time-independent analysis significantly overestimates the time-dependent hazard predictions by a factor of 4 . Such a large overestimation stems 

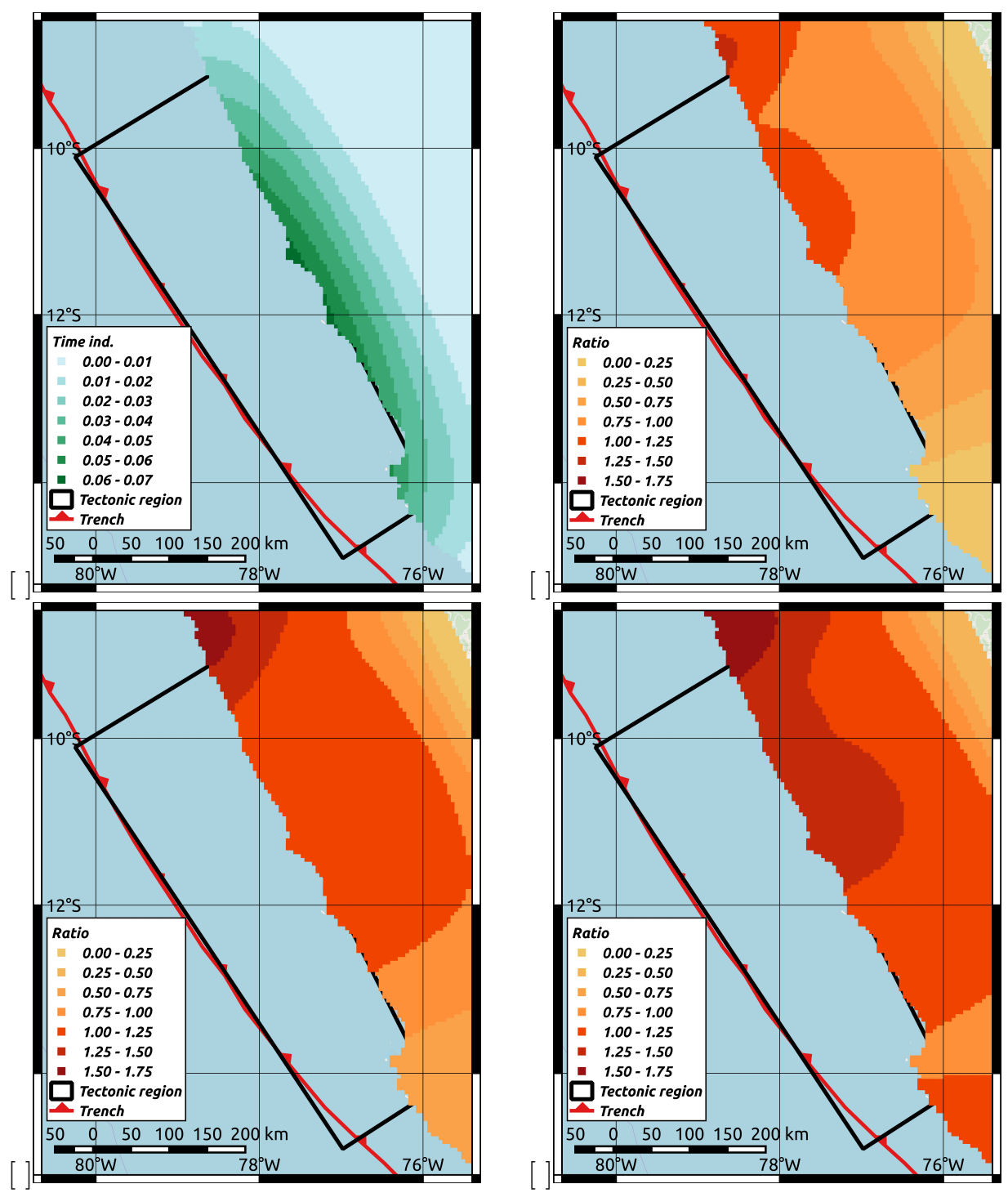

Figure 12: Comparison of $\mathrm{P}[\mathrm{PGA}>0.4 \mathrm{~g}]$ during 30 years: (a) time independent, (b), time dependent from 2018 to 2047 (c) time dependent with 30 extra years of seismic gap, (d) time dependent with 60 extra years of seismic gap.

from the fact that, unlike the time-independent analysis, the proposed model successfully incorporates in the analysis the recent release of stress, strain and energy caused by the 2007 earthquake, which ruptured the two southernmost fault sections. As shown in Figure, the sections reset their probability of rupture immediately after an earthquake in the time-dependent analysis, whereas such a probability remains constant in the time-independent analysis.

To assess the effect of the seismic gaps on the hazard, the probability of exceeding a PGA of $0.4 \mathrm{~g}$ from 2048 to 2077 was calculated with the assumption that the seismic gap is extended for 30 years. This assessment is equivalent to a what-if analysis that assumes that there will no be earthquake occurrences from 2018 to 2047 in the tectonic region. Figure shows the ratio between these time-dependent probabilities and those from the time-independent analysis. The comparison between Figures and shows how the hazard grows as the "seismic gap" increases. In the northern zone, the time-dependent hazard is larger than the time-independent hazard as a result of the stress and strain accumulation during the additional 30-year seismic gap. The increase in the hazard in the whole fault is driven by the increase of rupture probability in each individual sections. Once the rupture probabilities of the sections exceed the one of the exponential, as shown in Figure, the time-dependent hazard of the tectonic fault system will start exceeding the time-independent hazard.

Figure shows the ratio of time-dependent to independent hazard probabilities after including additional 30 years of seismic gap (i.e., the hazard is calculated from 2078 to 2107 assuming no earthquakes 
from 2018 to 2077). It can be seen that the hazard keeps increasing in the region. The northern region reaches hazard estimates $25 \%$ to $75 \%$ larger than those predicted with the time-independent analysis. Because of the BPT distribution, the probabilities of rupture occurrence at individual sections reach a constant plateau after long seismic gaps. After including the additional 30 years of seismic gap, most of the sections were close to their respective plateaus. For example, sections 4 and 5 were analyzed in the time interval between 104 and 134 years because the last rupture in those sections was in 1974. Figure shows that the rupture probability is practically the same in this time interval. Most of the sections are close to their respective plateaus; therefore, the regional hazard shown in Figure is approximately at the plateau of the time-dependent hazard in the region.

\section{CONCLUSIONS}

This paper presented a novel probabilistic formulation for modeling the space and time interactions of earthquake mainshocks in tectonic faults. The formulation (1) idealizes a tectonic fault area as a twodimensional surface, (2) discretizes the surface into small sections, and (3) models rupture occurrence of the section system as a correlated, multivariate Bernoulli process. The formulation models rupture occurrence of individual sections as Brownian passage-time (BPT) distributions, which are able to capture time-dependency of rupture occurrence (i.e., longer seismic gaps can increase the likelihood of rupture occurrence). Correlation models were also introduced to represent spatial interdependencies of rupture occurrences among the section of the fault, which captures the process of earthquake nucleation and rupture propagation among neighboring sections of the fault.

The model presented here is a suitable alternative for estimating seismic hazard to both probabilistic and physics-based existing models. Although physics-based models successfully capture complex features of rupture behavior, the uncertainty in the model parameters and the heavy computational demands make these models difficult to implement in full seismic hazard calculations. In contrast, while current probabilistic models do not capture as many complex features of rupture behavior, their simplicity and smaller computation times make them attractive for seismic hazard analysis. Thus, the proposed formulation is probabilistic and advances other existing probabilistic methods because it (1) captures time and space interactions of mainshocks, (2) preserves the marginal distribution of interarrival times after including the spatial rupture interaction in multiple sections, i.e., model consistency, and (3) has an implicit physical interpretation consistent with current modern earthquake rupture behavior theories. This paper has provided both a mathematical proof and demonstration through simulation for model consistency, as well as a simple approach for parameter estimation.

A case study demonstrating the applicability of the model presented here has also been provided. The study evaluated the rupture occurrence of large interface earthquakes in the subduction zone along the Coast of Lima, Peru. The historical catalog in this region contains earthquakes with magnitudes larger than 7.5 that occurred during the last 450 years in the region. Multiple parameters were tested to calibrate exponential and spherical correlograms to the historical catalog. The spherical correlogram performed better at reproducing the rupture correlations found in the historical catalog. However, the results show that both correlograms can be successfully adjusted to replicate fairly well the annual exceedance rates of magnitude occurrence and the spatial variations of average seismic moment release.

The suitability of the proposed model to represent the space and time interactions of earthquakes was evaluated through the AIC method. The proposed model was compared to a simplified model that is able to capture time interactions of earthquakes but not space interactions. Though the proposed model is more complex than the simplified model, the AIC results demonstrate that our model performs statistically better than the simplified model, increasing the likelihood of representing earthquake data and balancing the risk of model overfitting. Additional AIC results showed that the proposed model performs statistically better with the spherical correlogram than with the exponential correlogram.

The model was also used to calculate time-dependent seismic hazard resulting from the large ruptures in the earthquake data. The probability of peak ground accelerations (PGA) larger than $0.4 \mathrm{~g}$ during the next 30 years was estimated in the region. The results demonstrate that the proposed model was able to capture the spatial and temporal variations of earthquake occurrence stemming from the different mean interarrival times in the fault sections and the locations of recent earthquakes. In the application to Lima, the proposed model predicts similar hazard estimates $( \pm 25 \%)$ to the Poissonian time-independent analysis in the mid and northern regions. However, in the southern region, where a recent earthquake occurred, the seismic hazard was reduced to up to a fourth of the time-independent hazard, demonstrating that the proposed model successfully captures seismic gap effects. Additional results showed that if no 
large earthquake occurs in the fault during the next 30 years, the time-dependent seismic hazard will exceed the time-independent hazard in most of the region.

\section{Data and Resources}

The data and resources in this paper was subdivided in three folders in this link https://purl. stanford.edu/fk828tc8567. In the first folder, "Previous ruptures in GIS", the rupture areas of the earthquakes in 1940, 1966, 1974, and 2007 are provided in a GIS format. The boundaries of the tectonic region of analysis shown in black in Figure 4 are also provided in GIS format. In the second folder "Scripts", Matlab scripts to reproduce the rupture simulation are provided. In the third folder "Consistency Simulation", the file with six figures showing the consistency of the model in all fault sections for the spherical correlogram with six different $\gamma$ values can be found. 


\section{Acknowledgments}

The authors thank Prof. William Ellsworth, from the School of Earth, Energy, and Environmental Sciences at Stanford University, who provided insightful comments and feedback regarding the model proposed in the paper. The authors also appreciate the constructive comments and suggestions of the BSSA reviewers of the paper. This research was funded by the John A. Blume Research Fellowship and the Shah Family Fellowship through the Department of Civil and Environmental Engineering at Stanford University. Additionally, this research was partially supported by the NSF Grant 1645335. The authors are grateful for their generous support. 


\section{References}

Abrahamson, N., Gregor, N., and Addo, K. (2016). BC hydro ground motion prediction equations for subduction earthquakes. Earthquake Spectra, 32(1):23-44.

Akaike, H. (1974). A new look at the statistical model identification. IEEE Transactions on Automatic Control, 19(6):716-723.

Anagnos, T. and Kiremidjian, A. S. (1984). Stochastic Time-predictable Model for Earthquake Occurrences. Bulletin of the Seismological Society of America, 74(6):2593-2611.

Barbot, S., Lapusta, N., and Avouac, J.-p. (2012). Under the Hood of the Earthquake Machine: Toward Predictive Modeling of the Seismic Cylce: Supplementary Material. Science, 336(May):1-20.

Bozdogan, H. (1987). Model Selection and Akaike's Infomration Criterion (AIC): The General Theory and its Analytical Extensions. Psychometrika, 52(3):345-370.

Ceferino, L., Galvez, P., Ampuero, J.-P., Kiremidjian, A., and Deierlein, G. (2020). Bayesian Updating of Earthquake Rupture Model using Historic and Synthetic Physics-based Earthquake Catalogs. Bulletin of the Seismological Society of America, In Review.

Ceferino, L., Kiremidjian, A., and Deierlein, G. (2017). Space and time interaction modeling of earthquake rupture occurrence. In ICOSAAR.

Ceferino, L., Kiremidjian, A., and Deierlein, G. (2018). Parameter Estimation Methods for Modeling of Time and Space Interactions of Earthquake Rupture. In 16th European Conference on Earthquake Engineering, Thessaloniki.

Chhikara, A. R. S. and Folks, J. L. (1977). The Inverse Gaussian Distribution as a Lifetime Model. Technometrics, 19(4):461-468.

Chlieh, M., Perfettini, H., Tavera, H., Avouac, J. P., Remy, D., Nocquet, J. M., Rolandone, F., Bondoux, F., Gabalda, G., and Bonvalot, S. (2011). Interseismic coupling and seismic potential along the Central Andes subduction zone. Journal of Geophysical Research: Solid Earth, 116(12):1-21.

Cosentino, P., Ficarra, V., and Luzio, D. (1977). Truncated Exponential Frequency-Magnitude Relationship in Earthquake Statistics. 67(6):1615-1623.

Dieterich, J. (1994). A constitutive law for rate of earthquake its application to earthquake clustering production and Haberman, Alternate assumptions to ( 2 ) might be. Journal of Geophysical Research: Solid Earth, 99(B2):2601-2618.

Dieterich, J. H. (1979). Modeling of rock friction: 1. Experimental results and constituve equations. Journal of geophysical research, 84(9):2161-2168.

Dorbath, L., Cisternas, A., and Dorbath, C. (1990). Assessment of the size of large and great historical earthquakes in Peru. Bulletin of the Seismological Society of America, 80(3):551-576.

Ellsworth, W. L. and Beroza, G. C. (1995). Seismic Evidence for an Earthquake Nucleation. SCIENCENEW YORK THEN WASHINGTON, pages 851-851.

Field, E. H. (2015). Computing Elastic-Rebound-Motivated Earthquake Probabilities in Unsegmented Fault Models: A New Methodology Supported by Physics-Based Simulators. Bulletin of the Seismological Society of America, 105(2A):544-559.

Field, E. H., Biasi, G. P., Bird, P., Dawson, T. E., Felzer, K. R., Jackson, D. D., Johnson, K. M., Jordan, T. H., Madden, C., Michael, A. J., Milner, K. R., Page, M. T., Parsons, T., Powers, P. M., Shaw, B. E., Thatcher, W. R., Weldon, R. J., and Zeng, Y. (2015). Long-Term Time-Dependent Probabilities for the Third Uniform California Earthquake Rupture Forecast (UCERF3). Bulletin of the Seismological Society of America, 105(2A):511-543.

Field, E. H., Dawson, T. E., Felzer, K. R., Frankel, A. D., Gupta, V., Jordan, T. H., Parsons, T., Petersen, M. D., Stein, R. S., Weldon, R. J., and Wills, C. J. (2009). Uniform California earthquake rupture forecast, version 2 (UCERF 2). Bulletin of the Seismological Society of America, 99(4):2053-2107. 
Field, E. H. and Gupta, V. (2008). Conditional, Time-dependent probabilities for segmented Type-A faults in the WGCEP UCERF 2, Appendix N in The Uniform California Earthquake Rupture Forecast, version 2 (UCERF 2): U.S. Geological Survey Open-File Report 2007-1437N and California Geological. Technical report, United States Geological Survey, Pasadena, CA.

Field, E. H., Milner, K. R., Hardebeck, J. L., Page, M. T., van der Elst, N., Jordan, T. H., Michael, A. J., Shaw, B. E., and Werner, M. J. (2017). A spatiotemporal clustering model for the third uniform California earthquake rupture forecast (UCERF3-ETAS): Toward an operational earthquake forecast. Bulletin of the Seismological Society of America, 107(3):1049-1081.

Frankel, A. (1995). Mapping seismic hazard in the central and eastern United States. Seismological Research Letters, 66(4):8-21.

Galvez, P., Ampuero, J. P., Dalguer, L. A., Somala, S. N., and Nissen-Meyer, T. (2014). Dynamic earthquake rupture modelled with an unstructured 3-D spectral element method applied to the 2011 M9 Tohoku earthquake. Geophysical Journal International, 198(2):1222-1240.

Gardner, J. K. and Knopoff, L. (1974). Bulletin of the Seismological Society of America IS THE SEQUENCE OF EARTHQUAKES IN SOUTHERN CALIFORNIA, WITH AFTERSHOCKS REMOVED, POISSONIAN? Bulletin of the Seismological Society of America, 64(5):1363-1367.

Geller, R. J., Mulargia, F., and Stark, P. B. (2015). Why We Need a New Paradigm of Earthquake Occurrence. In Subduction Dynamics: From Mantle Flow to Mega Disasters, Geophysical Monograph 211, pages 183-191. John Wiley \& Sons, first edition.

Hagiwara, Y. (1974). Probability of earthquake occurrence as obtained from a Weibull distribution analysis of crustal strain. Tectonophysics, 23(3):313-318.

Helmstetter, A. and Werner, M. J. (2014). Adaptive smoothing of seismicity in time, space, and magnitude for time-dependent earthquake forecasts for California. Bulletin of the Seismological Society of America, 104(2):809-822.

Jin, R., Wang, S., Yan, F., and Zhu, J. (2015). Generating Spatial Correlated Binary Data Through a Copulas Method. 3(4):206-212.

Jones, L. M. (1994). Foreshocks, aftershocks, and earthquake probabilities: accounting for the Landers earthquake. Bulletin of the Seismological Society of America, 84(3):892-899.

Kagan, Y. Y. and Jackson, D. D. (1991). Seismic gap hypothesis: ten years after. Journal of Geophysical Research, 96(B13):419-431.

Kagan, Y. Y. and Jackson, D. D. (1999). Worldwide doublets of large shallow earthquakes. Bulletin of the Seismological Society of America, 89(5):1147-1155.

Kagan, Y. Y. and Jackson, D. D. (2014). Statistical earthquake focal mechanism forecasts. Geophysical Journal International, 197(1):620-629.

Kagan, Y. Y. and Jackson, D. D. (2015). Likelihood analysis of earthquake focal mechanism distributions. Geophysical Journal International, 201(3):1409-1415.

Kagan, Y. Y. and Knopoff, L. (1987). Random stress and earthquake statistics: time dependence. Geophysical Journal International, 88:723-731.

Kanamori, H. (1977). The energy release in great earthquakes. Journal of Geophysical Research, $82(20): 2981$.

Kelleher, J. a. (1972). Rupture zones of large South American earthquakes and some predictions. Journal of Geophysical Research, 77(11):2087.

Kiremidjian, A. S. and Anagnos, T. (1984). Stochastic Slip-predictable Model for Earthquake Occurrances. Bulletin of the Seismological Society of America, 74(2):739-755.

Langer, C. and Spence, W. (1995). The 1974 Peru Earthquake Series. Bulletin of the Seismological Society of America, 85(3):665-687. 
Lohman, R. B. and McGuire, J. J. (2007). Earthquake swarms driven by aseismic creep in the Salton Trough, California. Journal of Geophysical Research: Solid Earth, 112(4):1-10.

Luo, Y., Ampuero, J. P., Miyakoshi, K., and Irikura, K. (2017). Surface Rupture Effects on Earthquake Moment-Area Scaling Relations. Pure and Applied Geophysics, 174(9):3331-3342.

Lutz, K. A. and Kiremidjian, A. S. (1995). A Stochastic Model for Spatially and Temporally Dependent Earthquakes. Bulletin of the Seismological Society of America, 85(4):1177-1189.

Marone, C. (1998). Laboratory-Derived Friction Laws and Their Application To Seismic Faulting. Annual Review of Earth and Planetary Sciences, 26(1):643-696.

Marsan, D. and Lengliné, O. (2008). Extending earthquakes' reach through cascading. Science, 319(5866):1076-1079.

Matthews, M. V., Ellsworth, W. L., and Reasenberg, P. a. (2002). A Brownian model for recurrent earthquakes. Bulletin of the Seismological Society of America, 92(6):2233-2250.

Nishenko, S. and Buland, R. (1987). A generic recurrence interval distribution for earthquake forecasting. Bulletin of the Seismological Society of America, 77(4):1382-1399.

Ogata, Y. (1988). Statistical models for earthquake occurrences and residual analysis for point processes.

Ogata, Y. (1998). Space-time Point-process Models for Earthquake Occurrences. Annals of the Institute of Statistical Mathematics, 50(2):379-402.

Petersen, M. D., Moschetti, M. P., Powers, P. M., Mueller, C. S., Haller, K. M., Frankel, A. D., Zeng, Y., Rezaeian, S., Harmsen, S. C., Boyd, O. S., Field, N., Chen, R., Rukstales, K. S., Luco, N., Wheeler, R. L., Williams, R. A., and Olsen, A. H. (2014). Documentation for the 2014 Update of the National Seismic Hazard Maps. Technical report, USGS.

Poiata, N., Koketsu, K., and Miyake, H. (2010). Source processes of the 2009 Irian Jaya, Indonesia, earthquake doublet. Earth, Planets and Space, 62(5):475-481.

Reasenberg, P. (1985). Second-Order Moment of Central California Seismicity, 1969-1982. Journal of Geophysical Research, 90(B7):5479-5495.

Reid, H. F. (1911). The Elastic-Rebound Theory of Earthquakes. Bulletin of the Department of Geology, University of California Publications, vol. 6, no:413-444.

Rhoades, D. A. and Evison, F. F. (2004). Long-range earthquake forecasting with every earthquake a precursor according to scale. Pure and Applied Geophysics, 161(1):47-72.

Richards-Dinger, K. and Dieterich, J. H. (2012). RSQSim Earthquake Simulator. Seismological Research Letters, 83(6):983-990.

Rikitake, T. (1974). Probability of Earthquake Occurrence as Estimated from Crustal Strain. Tectonophysics, 23:299-312.

Ruina, A. (1983). Slip instability and state variable friction laws. Journal of Geophysical Research: Solid Earth, 88(B12):10359-10370.

Savage, J. C. and Langbein, J. (2008). Postearthquake relaxation after the 2004 M6 Parkfield, California, earthquake and rate-and-state friction. Journal of Geophysical Research: Solid Earth, 113(10):1-17.

Schwartz, D. P. and Coppersmith, K. J. (1984). Fault behavior and characteristic earthquakes: Examples from the Wasatch and San Andreas Fault Zones. Journal of Geophysical Research: Solid Earth, 89(B7):5681-5698.

Shaw, B. E. (1993). Generalized Omori law for aftershocks and foreshocks from a simple dynamics. Geophysical Research Letters, 20(10):907-910.

Shimazaki, K. and Nakata, T. (1980). Time-predictable recurrence model for large earthquakes. Geophysical Research Letters, 7(4):279-282. 
Silva, V., Crowley, H., Pagani, M., Monelli, D., and Pinho, R. (2014). Development of the OpenQuake engine, the Global Earthquake Model's open-source software for seismic risk assessment. Natural Hazards, 72(3):1409-1427.

Strader, A. and Jackson, D. D. (2014). Near-prospective test of Coulomb stress triggering. Journal of Geophysical Research: Solid Earth RESEARCH, 119:3064-3075.

Strader, A., Jackson, D. D., Hardebol, N. J., Maier, C., Nick, H., Geiger, S., Bertotti, G., Boro, H., Behn, M. D., Grove, T. L., Strader, A., Jackson, D. D., Behn, M. D., Grove, T. L., Strader, A., Jackson, D. D., Hardebol, N. J., Maier, C., Nick, H., Geiger, S., Bertotti, G., and Boro, H. (2015). Journal of Geophysical Research : Solid Earth. Journal of Geophysical Research: Solid Earth, 120:1667-1676.

Strasser, F. O., Arango, M., and Bommer, J. J. (2010). Scaling of the Source Dimensions of Interface and Intraslab Subduction-zone Earthquakes with Moment Magnitude. Seismological Research Letters, 81(6):951-954.

Tweedie, M. C. K. (1957). Statistical Properties of Inverse Gaussian Distribution I. The Annals of Mathematical Statistics, 28(2):362-377.

Udias, A. and Rice, J. (1975). Statistical analysis of microearthquake activity near San Andreas geophysical observatory, Hollister, California. Bulletin of the Seismological Society of America, 65(4):809-827.

Utsu, T. (1969). A Statistical Significance Test of the Difference in b-value between Two Earthquake Groups. Journal of Physics of the Earth, 14(2):37-40.

Villegas-Lanza, J. C., Chlieh, M., Cavalié, O., Tavera, H., Baby, P., Chire-Chira, J., and Nocquet, J.-M. (2016). Active tectonics of Peru: Heterogeneous interseismic coupling along the Nazca megathrust, rigid motion of the Peruvian Sliver, and Subandean shortening accomodation. Journal of Geophysical Research : Solid Earth, pages 1-24.

Wesnousky, S. G. (1994). The Gutenberg-Richter or characteristic earthquake distribution, which is it? Bulletin of the Seismological Society of America, 84(6):1940-1959.

Zheng, X. and Vere-Jones, D. (1991). Application of Stress and Release Models to Historical Earthquakes from North China. Pure and Applied Geophysics, 135(4).

Zheng, X. and Vere-Jones, D. (1994). Further applications of the stochastic stress release model to historical earthquake data. Tectonophysics, 229(1-2):101-121.

Zhuang, J., Harte, D., Werner, M. J., Hainzl, S., and Zhou, S. (2012). Basic models of seismicity: Temporal models. In Models and Techniques for Analyzing Seismicity, number August. Community Online Resource for Statistical Seismicity Analysis.

Zhuang, J., Werner, M. J., Harte, D., Hainzl, S., and Zhou, S. (2011). Basic models of seismicity: Spatiotemporal models. In Models and Techniques for Analyzing Seismicity, number July, pages 1-41. Community Online Resource for Statistical Seismicity Analysis. 


\section{Appendixes}

\section{A Proof of consistency of the model}

The proof for model consistency is provided here. Consistency is shown by demonstrating that the interarrival time preserves its initial BPT distribution at any section when the model spatial interactions are included. It will be shown that the CDF of the BPT distribution will be preserved at each integer year because the model was also presented for discrete year-based time intervals. A similar proof can be derived for any other time intervals. Without loss of generality, it is considered that the $j$-th section experienced a rupture at year $t=0$ (i.e., $X_{0}(j)=1$ ). The next interarrival time $\tau_{j}$ will be smaller than $Y$ years if and only if there is at least one rupture in the section during the following $Y$ years. This event set is equivalent to the union of events consisting of having the next first rupture at each possible year between 1 and $Y$ (i.e., $X_{t}(j)=1, X_{t-1}(j)=0, X_{t-2}(j)=0, \ldots, X_{1}(j)=0$, for any $\left.1 \leq y \leq Y\right)$. Therefore, the equality shown in Equation A1 holds.

$$
P\left[\tau_{j} \leq Y\right]=P\left[\cup_{t=1}^{Y}\left(X_{t}(j)=1, X_{t-1}(j)=0, \ldots X_{1}(j)=0 \mid X_{0}(j)=1\right)\right]
$$

Because elements of the event set are mutually exclusive, then, the probability of the union can be assessed as the sum of probabilities shown in Equation A2.

$$
P\left[\tau_{j} \leq Y\right]=\sum_{t=1}^{Y} P\left[X_{t}(j)=1, X_{t-1}(j)=0, \ldots X_{1}(j)=0 \mid X_{0}(j)=1\right]
$$

Additionally, $X_{0}(j)=1$ is equivalent to $T_{1}(j)=1$ since a rupture resets the time since the last earthquake (Equation 1). Therefore, adding $T_{1}(j)=1$ to the conditioning set does not change the final results as shown in Equation A3a. Furthermore, each event in the sum of Equation A2 represents the probability that the next rupture occurs during year $y$. This is equivalent to the event: $\left\{T_{1}(j)=\right.$ $\left.1, T_{2}(j)=2, \ldots, T_{y}(j)=y, T_{y+1}(j)=1\right\}$. Since both events are equivalent, then Equation A3b holds.

$$
\begin{gathered}
P\left[\tau_{j} \leq Y\right]=\sum_{t=1}^{Y} P\left[X_{t}(j)=1, X_{t-1}(j)=0, \ldots, X_{1}(j)=0 \mid X_{0}(j)=1, T_{1}(j)=1\right] \\
P\left[\tau_{j} \leq Y\right]=\sum_{t=1}^{Y} P\left[T_{t+1}(j)=1, X_{t}(j)=1, T_{t}(j)=t, X_{j}(t-1)=0, T_{t-1}(j)=t-1, \ldots,\right. \\
\left.X_{j}(1)=0, T_{1}(j)=1 \mid X_{j}(0)=1, T_{1}(j)=1\right]
\end{gathered}
$$

In addition, the set $\left\{T_{t+1}(j), X_{t}(j)\right\}$ is a Markov chain because it is independent of all the previous rupture history conditioned on the last step $\left\{T_{t}(j), X_{t-1}(j)\right\}$. This is because $\left\{T_{t}(j), X_{t-1}(j)\right\}$ contains all the required information to assess the probability of rupture in the section during the next year. Then, Equation A3b can be rewritten into Equation A4.

$$
\begin{gathered}
P\left[\tau_{j} \leq Y\right]=\sum_{t=1}^{Y} P\left[X_{t}(j)=1, T_{t+1}(j)=0 \mid X_{t-1}(j)=0, T(t)=t\right] \times \\
P\left[X_{t-1}(j)=0, T_{t}(j)=t \mid X_{t-2}(j)=0, T_{t-1}(j)=t-1\right] \times \ldots \\
P\left[X_{1}(j)=0, T_{2}(j)=2 \mid X_{0}(j)=1, T_{1}(j)=1\right]
\end{gathered}
$$

Next, $T_{t+1}(j)$ is dropped from the event $\left\{T_{t+1}(j), X_{t}(j) \mid X_{t-1}(j), T_{t}(j)\right\}$ since $T_{t}(j)=1$ and $X_{t}(j)=0$ are equivalent events given the conditional term. $X_{t-1}(j)$ is also dropped from the conditional probability since the $T_{t}(j)$ is the only information that is needed to evaluate the likelihood of $X_{t}(j)$ (Equation 2). Then, Equation A5 holds.

$$
\begin{gathered}
P\left[\tau_{j} \leq Y\right]=\sum_{t=1}^{Y} P\left[X_{t}(j)=1 \mid T_{t}(j)=t\right] \times P\left[X_{t-1}(j)=0 \mid T_{t-1}=t-1\right] \times \ldots \\
P\left[X_{2}(j)=0 \mid \tau_{2}(j)=2\right] \times P\left[X_{1}(j)=0 \mid T_{1}(j)=1\right]
\end{gathered}
$$


Equation A5 shows that the earthquake occurrence likelihood at section $j$ can be calculated as the sum of probabilities of mutually exclusive events representing that the next rupture occurs a given year from 1 to $Y$. Because each multiplicand in the sums represents the conditional (over time) marginal (over space) probability of an event with a rupture (or no rupture) at section $j$ as shown in Equation 3 , then Equation A6 is equivalent to Equation A5.

$$
P\left[\tau_{j} \leq Y\right]=\sum_{t=1}^{Y} p_{t}(j) \times\left(1-p_{t-1}(j)\right) \times \cdots \times\left(1-p_{2}(j)\right) \times\left(1-p_{1}(j)\right)
$$

Equations 3 and A6 show that these conditional marginal probabilities, and therefore $P\left[\tau_{j} \leq Y\right]$, can be calculated independently from the spatial rupture interactions with neighboring sections. Though ruptures at any time are sampled jointly in the entire fault according to Equation 1, the copula method constructs a multivariate normal distribution in Equation 9 that preserves the conditional marginal distributions. After constructing the covariance matrix $\boldsymbol{\Sigma}$, which has ones in the diagonal elements, the copula method models $\boldsymbol{Z}_{\boldsymbol{t}}$ with mean $\mathbf{0}$ and covariance matrix $\boldsymbol{\Sigma}$. Because $\boldsymbol{Z}_{\boldsymbol{t}}$ is a multivariate normal, then the marginal distribution of $Z_{t}(j)$ at section $j$ is a univariate normal distribution with mean 0 and variance 1 . It follows that $p_{t}(j)$ equals $\Phi\left[Z_{t}(j)\right]$ according to Equation 9, where $\Phi[$.$] is the standard$ normal CDF, therefore, the correlogram introduced in the model does not change the final conditional marginal probability of rupture because $p_{t}(j)=P\left[X_{t}(j)=1 \mid T_{t}(j)\right]=\Phi\left[Z_{t}(j)\right]$ at section $j$ regardless of the correlations in $\boldsymbol{\Sigma}$.

Turning back to Equation A5, $p_{t}(j)$ represents the probability of having a rupture during year $t$ at section $j$ given that there was no rupture during the last $t-1$ years. Thus, this multiplicand is equivalent to the probability that the interarrival time $\tau_{j}$ at section $j$ is less than or equal to $t$ given that $\tau_{j}$ is greater than $t-1$ (i.e., $\tau_{j} \leq t \mid \tau_{j}>t-1$ ). The next multiplicand $P\left[X_{t-1}(j)=0 \mid \tau_{j}=t-1\right]$ is equivalent to $P\left[\tau_{j}>t-1 \mid \tau_{j}>t-2\right]$ because $X_{t-1}(j)=0$ means that there is no rupture during year $t-1$. Using the same logic, all the multiplicands of Equation A5 were replaced by their equivalences as a function of $\tau_{j}$ as shown in Equation A7a. Then, using the Bayes's rule, the conditional probabilities in Equation A7a were rewritten as shown in Equation A7b. Note that the multiplication in Equation A7b simplifies to the expression in Equation A7c since the denominator equals the numerator of the next term and $P\left[\tau_{j} \leq 0\right]$ is $0 . \quad F(t)$ equals $P\left[\tau_{j} \leq t\right]$ and is the BPT CDF (Equation 5). Finally, the first term of the $t$ summand cancels out with the second term of the $t+1$ summand. It can be seen that the final expression is equivalent to the BPT CDF and that the model preserves the interarrival time distribution. Therefore, the model is consistent.

$$
\begin{aligned}
P\left[\tau_{j} \leq Y\right] & =\sum_{t=1}^{Y} P\left[\tau_{j} \leq t \mid \tau_{j}>t-1\right] \times P\left[\tau_{j}>t-1 \mid \tau_{j}>t-2\right] \times \ldots P\left[\tau_{j}>2 \mid \tau_{j}>1\right] \\
& \times P\left[\tau_{j}>1 \mid \tau_{j}>0\right] \\
P\left[\tau_{j} \leq Y\right] & =\sum_{h=1}^{Y} \frac{P\left[t-1<\tau_{j} \leq t\right]}{1-P\left[\tau_{j} \leq t-1\right]} \times \frac{1-P\left[\tau_{j} \leq t-1\right]}{1-P\left[\tau_{j} \leq t-2\right]} \times \ldots \frac{1-P\left[\tau_{j} \leq 2\right]}{1-P\left[\tau_{j} \leq 1\right]} \times \frac{1-P\left[\tau_{j} \leq 1\right]}{1-P\left[\tau_{j} \leq 0\right]} \\
P\left[\tau_{j} \leq Y\right] & =\sum_{t=1}^{Y} P\left[t-1<\tau_{j} \leq t\right]=\sum_{t=1}^{Y} F_{\tau_{j}}(t)-F_{\tau_{j}}(t-1)=F_{\tau_{j}}(Y)-F_{\tau_{j}}(0)=F_{\tau_{j}}(Y)
\end{aligned}
$$

\section{B Likelihood function for Model Performance Testing accord- ing to Akaike Information Criterion}

The Akaike Information Criterion (AIC) value requires the estimation of the likelihood function $\hat{L}$ (Equation 12). The following two subsections describe how to estimate $\hat{L}$ for the proposed earthquake model and for the simplified model.

\section{Likelihood Function for Proposed Model}

Ceferino et al. (2020) formulated the likelihood function $\hat{L}$ for the proposed model according to Equations $\mathrm{B} 1$ and B2: 


$$
\begin{gathered}
\hat{L}=\prod_{t=1}^{H} P_{\boldsymbol{\mu}, \boldsymbol{\alpha}, \gamma}\left[\boldsymbol{X}_{\boldsymbol{t}} \mid \boldsymbol{T}_{\boldsymbol{t}}\right] \\
P\left[\boldsymbol{X}_{\boldsymbol{t}} \mid \boldsymbol{T}_{\boldsymbol{t}}\right]=P\left[\cap_{j=1}^{N} A_{j}\right], \text { where }\left\{\begin{array}{l}
A_{j}=\left\{Z_{t}(j) \leq \Phi^{-1}\left(p_{t}(j)\right)\right\} \text { if } X_{t}(j)=1, \\
\text { or } A_{j}=\left\{Z_{t}(j)>\Phi^{-1}\left(p_{t}(j)\right)\right\} \text { otherwise }
\end{array}\right.
\end{gathered}
$$

where $H$ represents the total number of years in the earthquake catalog and $P_{\mu, \alpha, \gamma}[$.$] is the Multivari-$ ate Bernoulli distribution in Equation 2. The vectors $\boldsymbol{\mu}=\left\{\mu_{j} \mid \forall j=1, \ldots, N\right\}, \boldsymbol{\alpha}=\left\{\alpha_{j} \mid \forall j=1, \ldots, N\right\}$ and the parameter $\gamma$ represent all the parameters of the proposed model. Additionally, the elements of the vector $\boldsymbol{Z}_{\boldsymbol{t}}$ are given in Equation 9, which are correlated through either the exponential or spherical correlation as indicated in Equations 7 and 8.

\section{Likelihood Function for Simplified Model}

The likelihood function $\hat{L}$ of the simplified model is formulated here according to the Equation B3.

$$
\hat{L}=P_{\beta}[\boldsymbol{r}, \boldsymbol{m}] P_{\alpha, \mu}[\boldsymbol{\tau}]=P[\boldsymbol{r} \mid \boldsymbol{m}] P_{\beta}[\boldsymbol{m}] P_{\alpha, \mu}[\boldsymbol{\tau}]
$$

Because in the simplified model the interarrival times are independent from the earthquake magnitudes and locations, $\hat{L}$ can be estimated as the product of the probability $P_{\alpha, \mu}[\boldsymbol{\tau}]$ of observing the interarrival times and the joint probability $P_{\beta}[\boldsymbol{r}, \boldsymbol{M}]$ of magnitudes and locations, where $\boldsymbol{\tau}=\left\{\tau_{k} \mid \forall k=1, \ldots, n\right\}$, $\boldsymbol{m}=\left\{m_{k} \mid \forall k=1, \ldots, n\right\}, \boldsymbol{r}=\left\{r_{k} \mid \forall k=1, \ldots, n\right\}$ are the datasets of earthquake interarrival times, magnitudes and locations in the entire fault, respectively, and $n$ is the number of datapoints in the dataset.

$P_{\alpha, \mu}[\boldsymbol{\tau}]$ can be estimated as shown in Equation B4, where $f_{\alpha, \mu}\left(\tau_{k}\right)$ is the pdf of the BPT distribution in Equation 4, where only a single set of parameters $\mu$ and $\alpha$ are used to describe interarrival times in the entire fault.

$$
P_{\alpha, \mu}[\boldsymbol{\tau}]=\prod_{k=1}^{n} P_{\alpha, \mu}\left[\tau_{k}\right]=\prod_{k=1}^{n} f_{\alpha, \mu}\left(\tau_{k}\right)
$$

The magnitudes and locations were discretized into bins according to the number of sections in the proposed model in order to conduct a clean model comparison. Therefore, $P[\boldsymbol{r} \mid \boldsymbol{m}]$ can be estimated as shown in Equation B5 because earthquake locations are uniformly distributed across the entire fault, where $N_{l}$ and $N_{w}$ are the number of sections along the length and width of the entire fault, and $l[$.$] are$ $w[$.$] are functions that determine how many sections s_{l k}$ and $s_{w k}$ break along these respective directions due to the earthquake magnitude $m_{k}$. These geometric features can be obtained from empirical scaling laws (Strasser et al., 2010) and total length $L$ and width $W$ of the fault.

$$
P[\boldsymbol{r} \mid \boldsymbol{m}]=\prod_{k=1}^{n} \frac{1}{N_{l}-s_{l k}+1} \times \frac{1}{N_{w}-s_{w k}+1}, \quad \text { where } s_{l k}=l\left[m_{k}\right], s_{w k}=w\left[m_{k}\right]
$$

Finally, $P_{\beta}[\boldsymbol{m}]$ can be estimated as in Equation B6. The function $M[$.$] maps the number of sections$ that break due a given earthquake to the corresponding magnitude. $\Delta_{M_{-}}$evaluates the bin width between magnitude bin center $M\left[s_{l k} \times s_{w k}\right]$ of the earthquake magnitude $m_{k}$ and the next smaller magnitude bin centered at $M\left[\left(s_{l k}-1\right) \times\left(s_{w k}-1\right)\right]$. Similarly, $\Delta_{M_{+}}$evaluates the bin width between the magnitude bin of $m_{k}$ and the next larger bin centered at $M\left[\left(s_{l k}+1\right) \times\left(s_{w k}+1\right)\right]$.

$$
P_{\beta}[\boldsymbol{m}]=\prod_{k=1}^{n} P_{\beta}\left[M\left[s_{l k} \times s_{w k}\right]-\frac{\Delta_{M_{-}}}{2}<m_{k} \leq M\left[s_{l k} \times s_{w k}\right]+\frac{\Delta_{M_{+}}}{2}\right]
$$

The magnitude distribution can be estimated using the double truncated exponential distribution shown in Equation B7 (Cosentino et al., 1977).

$$
P_{\beta}[m \leq M]=\frac{1-\exp \left(-\beta\left(m-m_{\min }\right)\right)}{1-\exp \left(-\beta\left(m_{\max }-m_{\min }\right)\right)}, \quad \text { where } m_{\min } \leq m \leq m_{\max }
$$

